\title{
High Photodegradation Performance of ZnO Nanoparticles Supported on Porous Zeolite Na-a: Effects of ZnO Loading
}

\section{Lawrence Kioko Munguti ( $\square$ mungutimunlak@yahoo.com )}

University of the Free State (QwaQwa Campus) https://orcid.org/0000-0001-7640-7358

\section{Francis Birhanu Dejene}

University of the Free State (QwaQwa Campus),

\section{Research Article}

Keywords: ZnO, Zeolite, Photocatalytic, Nanocomposites

Posted Date: April 20th, 2021

DOI: https://doi.org/10.21203/rs.3.rs-430420/v1

License: (c) (i) This work is licensed under a Creative Commons Attribution 4.0 International License.

Read Full License 


\title{
High photodegradation performance of $\mathrm{ZnO}$ nanoparticles supported on porous Zeolite Na-A: Effects of ZnO loading
}

\author{
Lawrence Kioko Munguti $^{* 1,2}$, Francis Birhanu Dejene ${ }^{1}$ \\ 1. University of the Free State (QwaQwa Campus), Private Bag X13, Phuthaditjhaba 9866, \\ South Africa \\ 2. South Eastern Kenya University, P.O. Box 170-90200 Kitui-Kenya \\ * Corresponding author e-mail address: mungutimunlak@yahoo.com
}

\begin{abstract}
Zeolite Na-A supported $\mathrm{ZnO}$ nanocomposites ( $\mathrm{ZnO} /$ Zeolite $\mathrm{Na}-\mathrm{A} \mathrm{NCs}$ ) were synthesized at low temperature $\left(70^{\circ} \mathrm{C}\right)$ via the sol-gel process and characterized by X-ray diffraction technique (XRD), scanning electron microscopy (SEM), transmission electron microscopy (TEM), UV-visible diffuse reflectance spectroscopy (UV-vis DRS) and Fourier transform infrared (FTIR) spectroscopy for structural, morphological, optical and bonding properties. The textural properties and porosity were obtained by Brunauer-Emmett-Teller (BET) technique. The obtained XRD and microscopy results indicated that the obtained nanopowders were crystalline in nature and no collapse of the structure of zeolite Na-A. In addition, the synthesized $\mathrm{ZnO}$ nanoparticles occurred mainly on the surface of the zeolite support. It is clear that the zeolite supported $\mathrm{ZnO}$ nanoparticles were more dispersed as compared to the pure $\mathrm{ZnO}$ with improved porosity and high surface area. Photocatalytic activity for the $\mathrm{ZnO}$ /zeolite $\mathrm{Na}-\mathrm{A}$ was tremendously increased which was attributed to the synergetic combined effects of both $\mathrm{ZnO}$ and zeolite aluminosilicate network such as increased surface area $\left(\mathrm{S}_{\mathrm{BET}}\right)$, high adsorption and restrained charge recombination.
\end{abstract}

Keywords: ZnO; Zeolite; Photocatalytic; Nanocomposites

\section{Introduction}

Recycling of waste-water is one of the commonly used methods for alleviating the perennial water shortage in many parts of the world. Much of the well-known causes of water pollution include natural biological pollutants such as human and animal waste which harbours micro-organisms most of them been protozoa and bacteria. Some agricultural practices such as improper use and disposal of farm fertilizers and chemical sprays may also contribute towards pollution of water. However, the greatest challenge comes from heavy industrial use of water resulting in to wide spread pollution from industrial chemical wastes such as dyes, heavy oils, acids and bases. In order to get rid of these persistent pollutants, advanced oxidation process (AOPs) that are driven by powerful non-selective oxidizing radicals such as $\mathrm{OH}^{*}$ have been developed [1]. The advantage of the AOPs is that the have the ability to convert organic compounds to less toxic molecules and in an ideal situation it may lead to a complete mineralization of the pollutant molecules in to $\mathrm{CO}_{2}$ and $\mathrm{H}_{2} \mathrm{O}$ [2].

Among the AOPs techniques, semiconductor heterogeneous photo-oxidation catalysts have proven to be very efficient and low cost effective procedures for removal of stable harmful organic and inorganic compounds including inorganic dyes [3]. These heterogeneous photocatalyst are responsible for the triggering of the formation of the active oxidation radical through the absorption photon energy at ambient conditions without 
regeneration of counter harmful bi-products. The heterogeneous photocatalysts are also advantageous in that they can be recycled thus cutting the use of the material and hence the cost of water treatment. Among the semiconductor heterogeneous photocatalysts, $\mathrm{ZnO}$ is one the most popular in removal of organic molecules since it is effective, inexpensive and non-toxic [4]. More advantages include high photon absorption in the UV. However $\mathrm{ZnO}$ has inherent challenges such as being a wide bandgap material $(E g=3.37 \mathrm{eV})$, its absorption spectrum is limited. $\mathrm{ZnO}$ is usually unstable, dissociates easily in acidic medium, has poor adsorption and is prone to particle agglomeration. These aggregations and agglomerations minimise the active photocatalytic surface thus reducing the performance or some case leading to a complete loss of photocatalytic ability [5].

In order to circumvent such limitations, attempts have been made to immobilize the catalyst on a suitable solid support such as the zeolite network, fibre glass and carbon. In such situations, the $\mathrm{ZnO}$ particles are well embedded in the walls or surface of support material thus maintain a suitable distribution. The effective result is increased porosity $\mathrm{ZnO}$ in a $\mathrm{ZnO} /$ Support material. In addition to the improved porosity, catalyst recovery process is eased by use of the zeolite support [6]. Among the above mentioned $\mathrm{ZnO}$ support materials, zeolites are the most preferred because of their extraordinary structures, uniform pores and straight channels which leads to high adsorption properties. Its lattice discontinuity points creates the uncompensated negative charges which in conjunction with $\mathrm{OH}^{-}$and $\mathrm{H}_{2} \mathrm{O}$ leads to a unique ion exchange property [7]. Zeolites exist naturally or can be obtained synthetically and are classified according to their structure in to various forms such as A, X, Y, ZSM-5 and clinoptilolite. Among these, single phase zeolite-A are highly preferred because of their large cation exchange capacity, large number of acid sites due to its 8-ring, 6-ring, and 4- ring channel structure with the largest cavity dimension measuring $0.41 \mathrm{~nm}$ $\mathrm{x} 0.41 \mathrm{~nm}$. Besides zeolite-A has a significant absorption and excellent water holding capacity in its free channels [7]. Previously, immobilization of $\mathrm{ZnO}$ on zeolite has been studied by various researchers such as Hadjltaief et al., [8] who worked on $\mathrm{ZnO}$ supported on clay obtained through the sol-gel process. Based on their results, $\mathrm{ZnO}$ nanoparticles were well dispersed on the clay surface that led to improved photocatalytic performance. Jagannatha et al., [9] worked on $\mathrm{ZnO} /$ Zeolite nanocomposite for photocatalytic elimination of benzophenone and caffeine which revealed increased photodegradation of $>80 \%$ compared to that of zeolites $10 \%$ or pure $\mathrm{ZnO}$. Similar results pertaining zeo/ZnO were also reported by Marrero et al., [10]. This implies that immobilization of ZnO NPs on a zeolite support can effectively increase the surface area leading to increase in the exposed active sites for photodegradation. Zeolites further increase the photocatalytic activity unlike other forms of support material because the zeolite itself can enhance the photocatalytic efficiency by minimizing the electron/hole recombination. Moreover, the ${ }^{-}$ $\mathrm{OH}^{-}$radicals on the surface of $\mathrm{ZnO}$ can easily be transferred onto the zeolite surface. Even though zeolite supported $\mathrm{ZnO}$ has been previously studied, several aspects such as the effect of $\mathrm{pH}$, annealing temperature and type of precursors have not been exhaustively investigated. In this research work, we focus on $\mathrm{ZnO}$ nanoparticles synthesized via sol-gel technique and supported on zeolite type A (zeolite Na-A). The specific objectives of the study is to report on different $\mathrm{ZnO}$ loadings and its effects on the various properties of the $\mathrm{ZnO} /$ zeolite-A catalyst that contributes to increased photocatalytic performance. The $\mathrm{ZnO} /$ zeolite catalyst nanopowders were synthesized via a slightly modified sol-gel method and analyzed by XRD, SEM, TEM, BET and UV-vis DRS. The catalyst's pollutants 
removal ability was tested by the photodegradation of methylene blue dye as a model pollutant.

\section{Experimental details}

\subsection{Precursors, solvents and support material}

During the synthesis process, analytical grade zinc acetate dihydrate $\left[\mathrm{Zn}\left(\mathrm{CH}_{3} \mathrm{COO}\right)_{2} .2 \mathrm{H}_{2} \mathrm{O}\right] 99.9 \%$ pure (Merck) was used without further purification as the precursor for $\mathrm{ZnO}$ whereas 2-isopropanol was the solvent during the sol-gel process. Monoethanolamine (99\% pure) was used as a stabilizing agent during the hydrolysis stage. Further materials used in this work included synthetic zeolite Na-A supplied by SigmaAldrich with a chemical formula $\mathrm{Na}_{12}\left[\left(\mathrm{AlO}_{2}\right)_{12}\left(\mathrm{SiO}_{2}\right)_{12}\right] 27 \mathrm{H}_{2} \mathrm{O}$ and pore opening $4 \AA$ which was used as the support frame for the zeolite supported $\mathrm{ZnO}$. Methylene blue dye of molecular formula $\mathrm{C}_{16} \mathrm{H}_{18} \mathrm{ClN}_{3} \mathrm{Sx} \mathrm{nH}_{2} \mathrm{O}, \mathrm{n}=2-3$ (concentration $=20 \mathrm{mmol} / \mathrm{L}$ ) (Merck) was used as the model pollutant. Analytical grade reagents for the preparation and also scavenger studies included sodium hydroxide $(\mathrm{NaOH})$, potassium permanganate $\left(\mathrm{KMnO}_{4}\right)$, hydrogen peroxide $\left(\mathrm{H}_{2} \mathrm{O}_{2}\right)$ and ascorbic acid $\left(\mathrm{C}_{6} \mathrm{H}_{8} \mathrm{O}_{6}\right)$ were also supplied by Merck Chemicals.

\subsubsection{Preparation of $\mathrm{ZnO}$ sol}

A stock solution was prepared as follows: zinc acetate dihydrate, $6.29 \mathrm{~g}$, was dissolved in 2-isopropanol $(100 \mathrm{ml})$ by magnetic stirring for 30 minutes inside a beaker. This was followed by addition of $1.73 \mathrm{ml}$ of Monoethanolamine drop-wise until a clear solution was achieved then followed by further stirring for 1 hour at $70{ }^{\circ} \mathrm{C}$. Monoethanolamine was used as a stabilizing agent. $\mathrm{ZnO}$ sols of varying $\% \mathrm{ZnO}(0.5,0.75,1.0,1.5$, and 2.0) concentrations were prepared from this stock solution.

\subsubsection{Preparation of the $\mathrm{ZnO} / \mathrm{Zeolite} \mathrm{Na}-\mathrm{A} \mathrm{NCs}$}

The procedure applied for the $\mathrm{Zn}^{2+}$ ion exchange in zeolite was obtained with a slight modification from [11] and was as follows: $2 \mathrm{~g}$ of zeolite powder was added to distilled water $50 \mathrm{ml}$ to form aqueous suspension then added to $0.5 \mathrm{M}$ of zinc acetate solution. The mixture was refluxed for 8 hours at a temperature of $80^{\circ} \mathrm{C}$ so as to allow ion exchange into and out of the zeolite framework then $\mathrm{ZnO}$ sol prepared in different concentrations as per the procedure in 2.1.1 above was added. $0.1 \mathrm{M}$ of sodium hydroxide $(\mathrm{NaOH})$ was added to the mixture up to a $\mathrm{pH}$ of 12 and resulting slurry was stirred for 3 hours at $70{ }^{\circ} \mathrm{C}$ then followed by ageing for 48 hours and drying at $100{ }^{\circ} \mathrm{C}$ for 4 hours. In order to immobilize $\mathrm{ZnO}$ on the zeolite calcination was the done at $600{ }^{\circ} \mathrm{C}$ for 4 hours. The obtained $\mathrm{ZnO} /$ Zeolite Na-A catalyst samples were labelled $(0.5 \%, 0.75 \%, 1.0 \%, 1.5 \%$ and $2.0 \%)$ $\mathrm{ZnO} /$ Zeolite Na-A respectively and were compared to both bare Zeolite and pure $\mathrm{ZnO}$ nanoparticles.

\subsection{Characterization and data collection methods}

\subsubsection{X-ray diffraction technique}

The structural characterization of the prepared zeo, $\mathrm{ZnO}$ and zeo/ZnO catalyst was carried out using Bruker AXS Discover diffractometer with monochromatic $\mathrm{CuK}_{\alpha}(1.5406 \AA)$ radiation. The powder samples were spread on low background $\mathrm{Si}$ sample holders, 
compacted and carefully placed on the sample stage then scanned for two-theta range $20^{\circ} \leq$ $2 \theta \leq 70^{\circ}$.

\subsubsection{Electron microscopy imaging}

The surface morphology and the effects of $\mathrm{ZnO}$ on the zeolite surface were observed using the Shimadzu Superscan ZU SSX-550 electron microscope. The scan was done at various points of the sample at different magnifications with the probe size of $115 \mathrm{~nm}$, probe current $0.02 \mathrm{nA}$ and accelerating voltage of $5.0 \mathrm{keV}$. Carbon coating was used during SEM measurement to prevent charging. EDX spectra of the respective samples was performed using an Oxford X-Max detector and the associated Aztec software. Beam setting were as follows: $15 \mathrm{KV}, 5 \mathrm{~mm}$ working distance and $120 \mathrm{~mm}$ aperture. TEM images were obtained using the Phillips 301 HRG Transmission electron microscope.

\subsubsection{UV-Vis diffuse reflectance spectra (DRS)}

The UV-vis diffuse reflectance spectra (DRS) were measured by the Perkin Elmer Lambda $950 \mathrm{UV}-\mathrm{Vis}-\mathrm{NIR}$ spectrophotometer within the wavelength $250 \mathrm{~nm}-800 \mathrm{~nm}$ equipped with an IRS 240 integrating sphere attachment. The reference spectrum was obtained from the reflectance of $\mathrm{BaSO}_{4}$ as the standard reflectance.

\subsubsection{Fourier transform infra-red (FT-IR)}

The characteristic functional groups were obtained by the Fourier transform infra-red (FTIR) spectrometer for all samples in the range $4000-400 \mathrm{~cm}^{-1}$. To obtain the infrared spectra, $0.02 \mathrm{~g}$ of the nanocomposite powder sample was mixed with dried potassium bromide (dried overnight in an oven for 24-hours) in the ratio of sample: $\mathrm{KBr}=1: 200$ pressed into pellets using mortar, pestle and other mechanical devices. The FTIR spectrum for pure $\mathrm{KBr}$ was also measured and used as the reference.

\subsubsection{Nitrogen adsorption-desorption}

Nitrogen adsorption-desorption isotherms were obtained at $77 \mathrm{~K}$ as a function of relative pressure $\mathrm{P} / \mathrm{P}_{0}$ for the range of 0.01-0.99 using a using the Micromeritics Tristar II 3020 (U.S.A.) Surface Area Analyzer. The out gassing of the samples was carried out at a temperature of $150{ }^{\circ} \mathrm{C}$ for $2 \mathrm{~h}$ under vacuum prior to each of the measurements. Specific surface area of some of the samples was calculated according to the Brunauer-EmmettTeller (BET) method using adsorption data in the relative pressure $\mathrm{P} / \mathrm{P}_{0}$ for the range 0.05 0.2 . The total pore volumes $\left(\mathrm{V}_{\mathrm{t}}\right)$ were estimated from the amount adsorbed at the relative pressure of 0.98 . The pore size distribution (PSD) was calculated by using the improved Barrett-Joyner-Halenda (BJH) model evaluated from the desorption branch.

\subsection{Photodegradation experiments}

\subsubsection{Photodegradation tests}

To test the photocatalytic performance of the synthesized zeolite, $\mathrm{ZnO}$ and zeo/ $\mathrm{ZnO}$ nanocomposites, photodegradation of methylene blue dye under ultraviolet light was conducted. An aqueous solution of methylene blue dye of concentration $20 \mathrm{mmol} / \mathrm{L}$ as the initial solution was prepared in a glass beaker and $0.03 \mathrm{~g}$ of each the different component photocatalyst incorporated into a $60 \mathrm{ml}$ of the aqueous solution followed by magnetic 
stirring in darkness to obtain desorption-adsorption equilibrium of MB molecules on the catalyst surface. Aliquots were taken after every one hour for 6 hours, and the intensity of the absorbance peak determined. The suspension was then subjected to a constant magnetic stirring at a temperature of $25^{\circ} \mathrm{C}$ under continuous illumination using the ultraviolet light. The source of the radiation was a low-pressure lamp emitting UV light at a wavelength of $365 \mathrm{~nm}$ positioned at about $12 \mathrm{~cm}$ above the photoreactor in open air conditions to avoid heating. The intensity of the emitted radiation was $413 \mathrm{~mW} / \mathrm{cm}^{2}$. An aliquot of $4 \mathrm{ml}$ was again taken after every one hour centrifuged and the catalyst filtered off, then the absorbance spectra of the supernatant measured using the UV-visible spectrometer and the intensity of the main absorption peak was recorded. The degradation efficiency of methylene blue dye was determined using the relation:

$$
\text { Degradation efficiency }(\%)=\frac{\left(C_{o}-C_{t}\right)}{C_{o}} \times 100
$$

Where $\mathrm{C}_{\mathrm{o}}$ and $\mathrm{C}_{\mathrm{t}}$ are the initial and final concentration of the MB dye.

\subsubsection{Degradation kinetics}

The photodegradation kinetics of methylene blue by the catalysts was fitted by the Langmuir-Hinshelwood model assuming that pollutants concentration is very low (usually a few dozen parts per million is common) $\mathrm{KC}<<1$, decomposition reaction assumes a pseudo-first-order kinetic model with respect to the target pollutant concentration.

Eq. (2) below represents the dependence between the dye concentrations in the aqueous versus time of UV irradiation.

$$
r_{t}=-\frac{d C_{t}}{d t}=\frac{k_{r} K_{1} C_{t}}{K_{2} C_{s t}+1+K_{1} C_{t}}
$$

$\mathrm{r}_{\mathrm{t}}$ is the reaction rate, $\mathrm{C}_{\mathrm{t}}$ is the concentration of the methylene blue dye at time $t, \mathrm{k}_{\mathrm{r}}$ is the reaction rate constant, $\mathrm{K}_{1}$ is the coefficient of adsorption of the dye, $\mathrm{K}_{2}$ is the adsorption coefficient of dissolvent and $\mathrm{C}_{\mathrm{st}}$ is the concentration of the solvent at time $\mathrm{t}$.

Compared with the reactant, the adsorption of the dissolvent is negligible. Hence, Eq. (2) can be expressed as Eq. (3).

$$
r_{t}=-\frac{d C_{t}}{d t}=\frac{k_{r} K_{1} C_{t}}{1+K_{1} C_{t}}
$$

It can be noted that the concentration of the dyes is extremely low and $\mathrm{K}_{1} \mathrm{C}_{\mathrm{t}}$ is much lower than 1. This implies that Eq. (3) can be further simplified to Eq. (4), where $\mathrm{K}$ is the product of $\mathrm{k}_{\mathrm{r}}$ and $\mathrm{K}_{1}$ and is also a constant.

$$
\mathrm{r}_{\mathrm{t}}=-\frac{\mathrm{d} \mathrm{C}_{\mathrm{t}}}{\mathrm{dt}}=\mathrm{k}_{\mathrm{r}} \mathrm{K}_{1} \mathrm{c}_{\mathrm{t}}=\mathrm{kc}_{\mathrm{t}}
$$

The final kinetic model (Eq. (6)) can be obtained by computing the integral of Eq. (4) to obtain Eq. 5. As seen in Eq. (6), the constant k represents the efficiency in the degradation of the MB dyes. 


$$
\begin{aligned}
& -\int_{\mathrm{c}_{\mathrm{o}}}^{\mathrm{c}_{\mathrm{t}}} \frac{1}{\mathrm{c}_{\mathrm{t}}} \mathrm{dt}=\int_{0}^{\mathrm{t}} \mathrm{kdt} \\
& -\ln \left(\frac{C_{o}}{C_{t}}\right)=\mathrm{kt}
\end{aligned}
$$

In Eq. 6, $\ln$ is the natural logarithm, $C_{o}$ is the target pollutants' (MB) initial concentration while $C_{t}$ is the concentration after time $t$ and $\mathrm{k}$ is the reaction rate constant. The nature of the dye degradation process is assumed to be of the first-order reaction, hence the constant reaction rate can be determined as the slope of the linear regression. The constant $\mathrm{k}$ can be obtained from the slope of the straight-line plot of $\ln \left(\mathrm{C}_{\mathrm{o}} / \mathrm{C}\right)$ versus $t$ in equation (6) as a function of experimental parameters.

\subsubsection{Stability tests}

Reusability is an important factor to consider in the application process of a catalyst as it greatly leads to cost reduction especially in long run or in case of large scale use in degradation systems. The regeneration experiments were performed and the photocatalysts were recycled 5 times. The regeneration of the composite in each subsequent cycle was done by washing with distilled water $(20 \mathrm{~mL})$, filtered, and then, the powder was dried at $100{ }^{\circ} \mathrm{C}$ for $3 \mathrm{~h}$.

\subsubsection{Scavenger studies}

In order to determine the participating radicals in the photodegradation process scavenger studies were carried using potassium iodide, isopropanol and ascorbic acid as hole $\left(h^{+}\right)$, hydroxide radical $\left(\mathrm{OH}^{\bullet}\right)$ and superoxide $\left(\mathrm{O}_{2}^{\bullet-}\right)$ radical hunters respectively. Effects of hydrogen peroxide on the rate of photodegradation of the catalyst was also studied.

\section{Results and discussion}

\subsection{Structural analysis of the zeo, $\mathrm{ZnO}$ and $\mathrm{ZnO} / \mathrm{Zeolite} \mathrm{Na}-\mathrm{A}$ composites 3.1.1 XRD patterns}

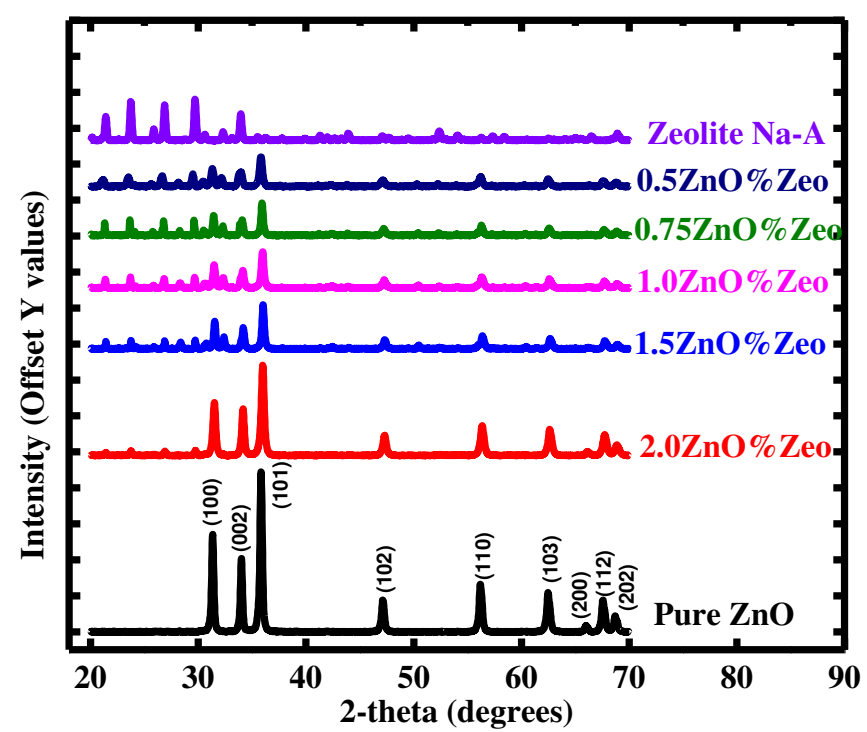

Fig. 1: XRD patterns for zeolites, $\mathrm{ZnO}$ and various \%loadings of $\mathrm{ZnO} / \mathrm{Zeolite} \mathrm{Na}-\mathrm{A}$ 
The microstructure of zeolites, pure $\mathrm{ZnO}$ and zeolite with varying loads of $\mathrm{ZnO}$ was obtained from the analyses of the obtained XRD diffraction patterns shown in figure 1 . The strong intense peaks obtained were a reflection of high crystallinity of the synthesised nanopowders. Based on the highest XRD intensity peaks, the zeolite peaks were indexed to $21.2,23.5,25.8,26.9,29.8,33.8$ two- theta degrees of the characteristic zeolite Na-A JCPDS card number (30-0222). On the other hand, the $\mathrm{ZnO}$ peaks where traced to JCPDS card number 36-1451 indexed to the hexagonal wurtzite structure with characteristic twotheta reflection $2 \theta=31.7^{\circ}, 34.2^{\circ}, 36.2^{\circ}, 47.5^{\circ}, 56.6^{\circ}, 62.9^{\circ}, 66.4^{\circ}, 68.0^{\circ} \& 69.0^{\circ}$. These peaks are associated with reflection planes (100), (002), (101), (102), (110), (103), (200), (112) \& (201) which crystallites in $\mathrm{P6}_{3} m c$ space group. The XRD structural patterns $\mathrm{ZnO}$ loaded zeolites revealed a combination of sharp peaks indexed to both $\mathrm{ZnO}$ and the zeolites indicating that the $\mathrm{ZnO} /$ Zeolite Na-A catalyst was also highly crystalline in nature. No impurity peaks were detected in the patterns indicating high purity of the synthesised samples. It was also clear that there was no collapse of the zeolite structure even after increased $\mathrm{ZnO}$ loadings. There was a redshift of $\mathrm{ZnO} /$ Zeolite peaks relative to the raw $\mathrm{ZnO}$ peaks which is an indicator of successful loading of $\mathrm{ZnO}$ on the zeolite. The shift may have been caused by compression of the zeolite structure by $\mathrm{ZnO}$ nanoparticle loading without changing its cubic morphology [11]. The intensity of Zeolite $\mathrm{Na}-\mathrm{A}$ peaks after $\mathrm{ZnO}$ loading appeared diminished mainly due to the shielding effect of the X-rays on the zeolite crystallites by the $\mathrm{ZnO}$ nanoparticles on the zeolite surface. Moreover, decreased XRD peak intensities implies that the ZnO NPs were also formed on the surface of the zeolites. This was further confirmed by the fact that increase in $\mathrm{ZnO}$ loading led to a decrease in the zeolite peaks and was in agreement with the obtained SEM images. Another possible cause of decreased zeolite peaks may be due reduced crystallinity caused by either base leaching of $\mathrm{Al}$ during modification or during the annealing process [12]. With no extra peaks, a match between $\mathrm{ZnO} /$ ZeolitesNa-A and the unloaded zeolite peak positions was observed which implies that the zeolite's internal framework was unaltered during the ion exchange and also after the $\mathrm{ZnO}$ loading. The obtained results agreed with those obtained by Ejhieh and Khorsandi [13].

\subsubsection{Crystalite size calculations by Scherrer}

The crystallite size of the $\mathrm{ZnO} / \mathrm{ZeoliteNa}-\mathrm{A}$ nanopowders, were calculated using the scherrer relation in equation 6 .

$$
\mathrm{D}=\frac{\mathrm{K} \cdot \lambda}{\beta \cdot \operatorname{Cos} \theta}
$$

Where $\mathrm{D}$ is the crystallite size, $\theta$ is the Braggs angle, $K$ is the Scherrer's constant $(K=$ $0.9), \lambda$ is the $X$-ray wavelength $(\lambda=1.5406 \AA)$ and $\beta$ is the Full Width at Half Maximum (FWHM) of the integral breadth of the most intense XRD peak.

The obtained crystallite sizes for $\mathrm{ZnO}$ ranged between 24.8 - $35.1 \mathrm{~nm}$ which indicates that the synthesized $\mathrm{ZnO} / \mathrm{Zeolite} \mathrm{Na}-\mathrm{A}$ reached the expected nanosize confirming a successful formation of the catalyst. Compared to the Zeolite Na-A average pore size of $81.12 \AA$, it is not possible that the $\mathrm{ZnO}$ crystallites could enter in to the zeolite micropores. This agrees with the obtained XRD results that the synthesized $\mathrm{ZnO}$ nanoparticles were only formed on the zeolite surface. It was also observed that crystallite sizes increased with the $\mathrm{ZnO}$ 
loading which is probably caused by high amount of $\mathrm{Zn}^{+}$ions due to increased precursor concentration.

Table 1: Effect of loading on $\mathrm{ZnO}$ crystallite size and spacing.

\begin{tabular}{|c|c|c|c|c|c|c|c|}
\hline \multirow[t]{2}{*}{ Sample } & \multirow[t]{2}{*}{$2 \theta^{\circ}$} & \multirow{2}{*}{$\begin{array}{l}\text { FWHM } \\
\text { radians }\end{array}$} & \multirow{2}{*}{$\begin{array}{l}\text { D-size } \\
(\mathrm{nm})\end{array}$} & \multirow{2}{*}{$\begin{array}{l}\text { d-spacing } \\
(\mathrm{nm})\end{array}$} & & \multicolumn{2}{|c|}{ Lattice constants } \\
\hline & & & & & & $\mathrm{a}(\AA ̊)$ & $c(\AA)$ \\
\hline & & & & & JCPDS & 3.249 & 5.206 \\
\hline $0.5 \% \mathrm{ZnO} / \mathrm{Zeolite} \mathrm{Na}-\mathrm{A}$ & 35.8174 & 0.00587 & 24.8 & 0.24455 & & 2.824 & 4.891 \\
\hline $0.75 \% \mathrm{ZnO} /$ Zeolite $\mathrm{Na}-$ & 35.9166 & 0.00449 & 26.5 & 0.2439 & & & \\
\hline A & & & & & & 2.816 & 4.878 \\
\hline $1.0 \% \mathrm{ZnO} / \mathrm{Zeolite} \mathrm{Na}-\mathrm{A}$ & 35.9761 & 0.00484 & 30.1 & 0.24352 & & 2.812 & 4.870 \\
\hline $1.5 \% \mathrm{ZnO} / \mathrm{Zeolite} \mathrm{Na}-\mathrm{A}$ & 35.996 & 0.00449 & 32.5 & 0.24338 & & 2.810 & 4.868 \\
\hline 2.0\% ZnO/Zeolite Na-A & 35.9761 & 0.00553 & 26.4 & 0.24352 & & 2.812 & 4.870 \\
\hline Pure $\mathrm{ZnO}$ & 35.8174 & 0.00414 & 35.1 & 0.24455 & & 2.824 & 4.891 \\
\hline
\end{tabular}

\subsubsection{Effects of loading on $\mathrm{ZnO}$ lattice constants and the induced strain}

According to Table 1, the prepared $\mathrm{ZnO}$ reported lattice constants slighltly smaller than the standard (JCPDS) values which could be due to nature of the synthesis technique as opposed to effects of the support. However there was a slight variation but not significant effect of the support on the obtained lattice constants which was caused by the induced lattice micro-strain.

The induced strain resulting from the loading of $\mathrm{ZnO}$ on the zeolite support was estimated by the Williamson-Hall plot as shown figure 2(a-f). The obtained values of microstrain are depicted on the plots of strain versus the $\mathrm{ZnO}$ loading in figure $2(\mathrm{~g})$ which reveals that the strain increased with loading up to a maximum of $1.5 \mathrm{ZZ}$ and then decreased. From the obtained values it is clear that the obtained strain had minimum impact on the internal structural changes induced by the zeolite support.
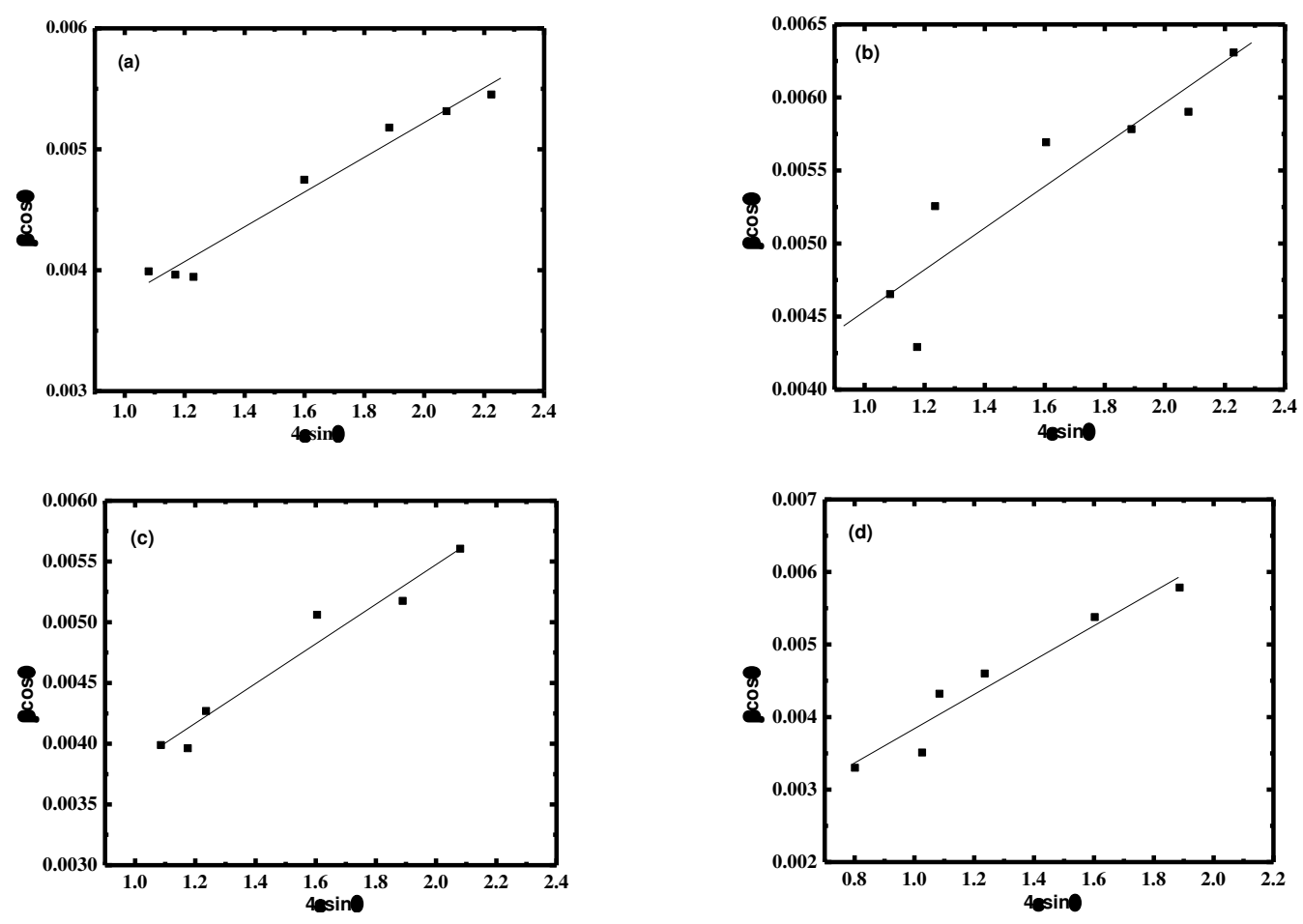

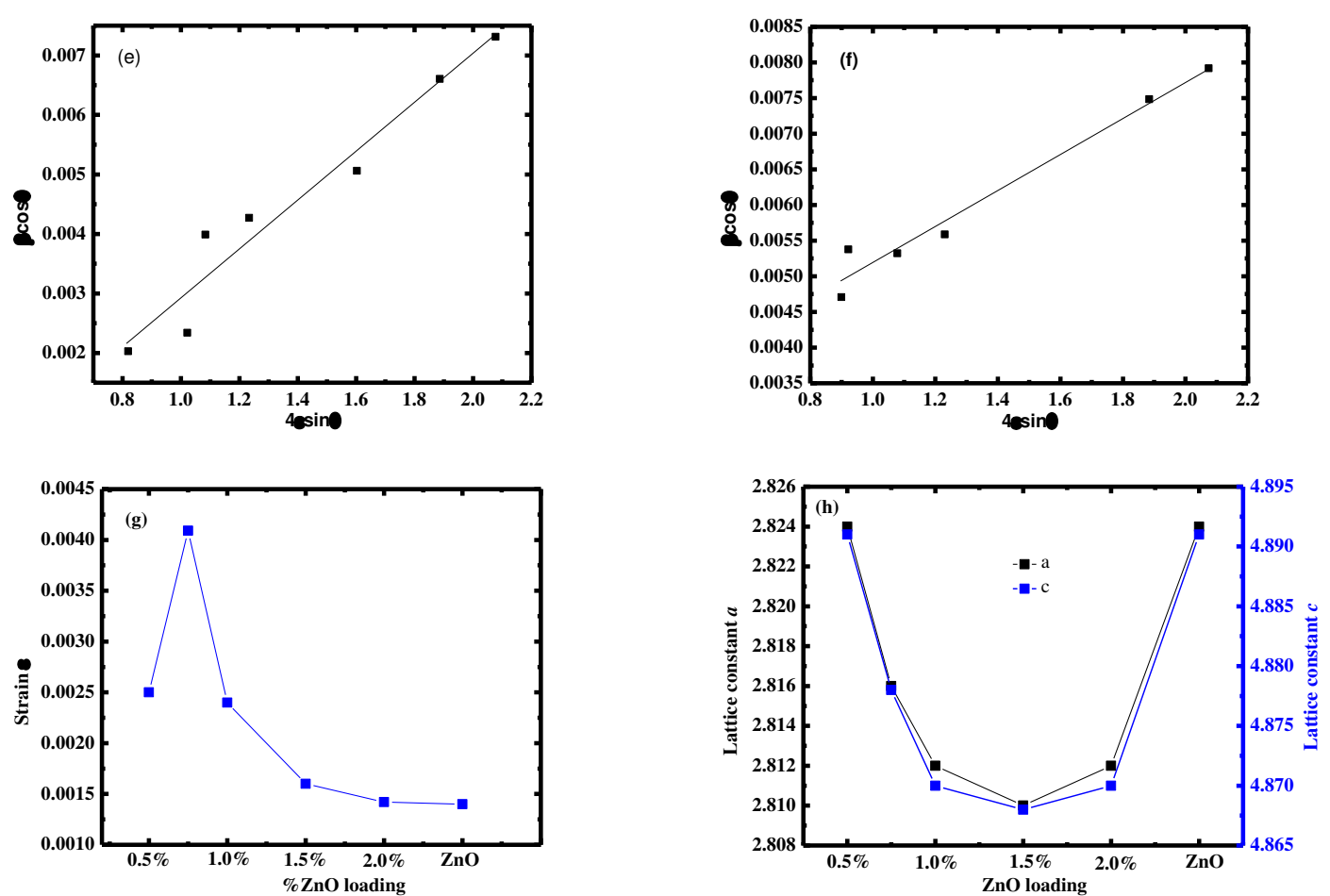

Fig. 2: Evaluation of strain by Williamson-Hall plots 2(a-f) whereas (g) and (h) shows the relationship between microstrain and lattice constants versus $\mathrm{ZnO}$ loading respectively.

\subsection{Morphological properties}

\subsubsection{SEM images}

The scanning electron microscope images obtained for zeo, $\mathrm{ZnO}$ and $\mathrm{ZnO} / \mathrm{Zeolite} \mathrm{Na}-\mathrm{A}$ catalyst are shown in fig. $3(\mathrm{a}-\mathrm{c})$. It is clear that both raw and ion-exchanged zeolites exhibited a cubic morphology with smooth surfaces typical for zeolite Na-A which implies that there was no structural changes induced during the ion-exchange process. On the other hand, $\mathrm{ZnO}$ nanoparticles maintained the granular spherical morphology dominated by particle agglomerations. The SEM images for the $\mathrm{ZnO} / \mathrm{Zeolite} \mathrm{Na}-\mathrm{A}$ indicated regions of different colour intensities with light grey representing the $\mathrm{ZnO}$ nanoparticles on a relatively darker zeolite background. In addition, the $\mathrm{ZnO}$ loaded zeolite composite maintained its cubic morphology which implies that there was no collapse of zeolite structure due to $\mathrm{ZnO}$ loading which is in agreement with the obtained the obtained XRD structural results. $\mathrm{ZnO}$ nanoparticles were observed attached to the zeolite surface. The attachment of the $\mathrm{ZnO}$ nanoparticles on the surface of the zeolite support is vital because it reduces the level of nanoparticle agglomerations and thus increases the exposed surface for photodegradation process. However the degree of agglomeration appeared to decrease with $\mathrm{ZnO}$ loading up to $1.5 \% \mathrm{ZnO}$ beyond which agglomerations increased attributed to excess ZnO. Similar SEM images were also obtained by Alswata et al.,[11] who studied zeolite supported $\mathrm{ZnO}$ Oxide nanocomposites for toxic metals removal from water. 

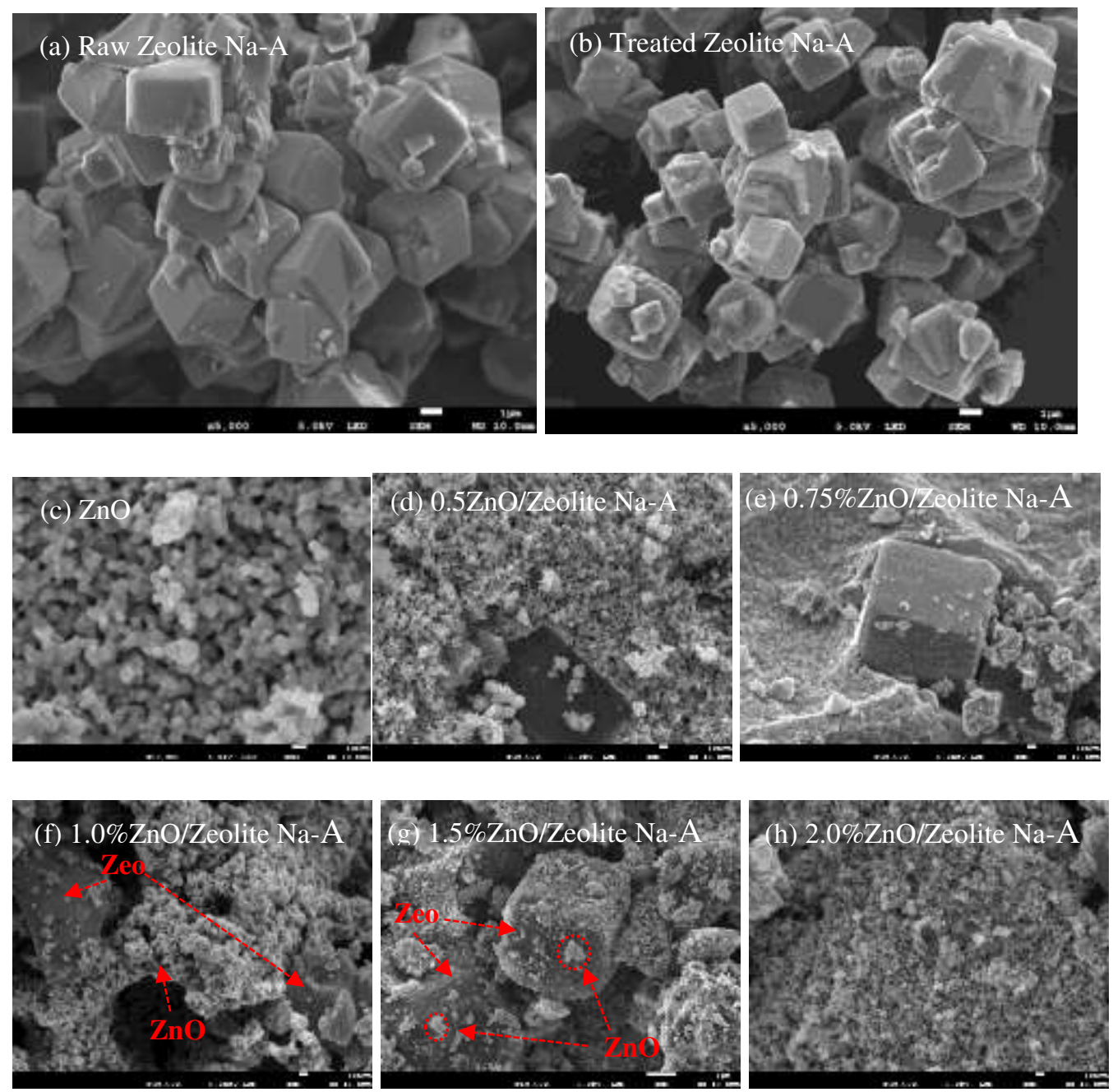

Fig. 3: SEM images for different $\mathrm{ZnO}$ loaded zeolite $\mathrm{Na}-\mathrm{A}$

\subsubsection{Elemental composition}

The EDX spectra for the elemental composition of pure $\mathrm{ZnO}$ and $\mathrm{ZnO} / \mathrm{Zeolite} \mathrm{Na}-\mathrm{A}$ nanocomposites is shown in figure 4. The spectra for pure $\mathrm{ZnO}$ oxide consisted of Zinc and Oxygen elements only and no impurity atoms were detected. In addition to the elements detected in pure $\mathrm{ZnO}$ spectrum, $\mathrm{ZnO} / \mathrm{Zeolite} \mathrm{Na}-\mathrm{A}$ reveled elements such as sodium $(\mathrm{Na})$, silicon $(\mathrm{Si})$ and aluminum $(\mathrm{Al})$ inherent in zeolites $\mathrm{Na}-\mathrm{A}$. This implies that $\mathrm{ZnO}$ was successfully loaded on zeolites. The carbon peak seen in the spectra of figure 4 (b) emanated from the carbon tape coating used during SEM imaging as explained in section 2.2.2 above. 

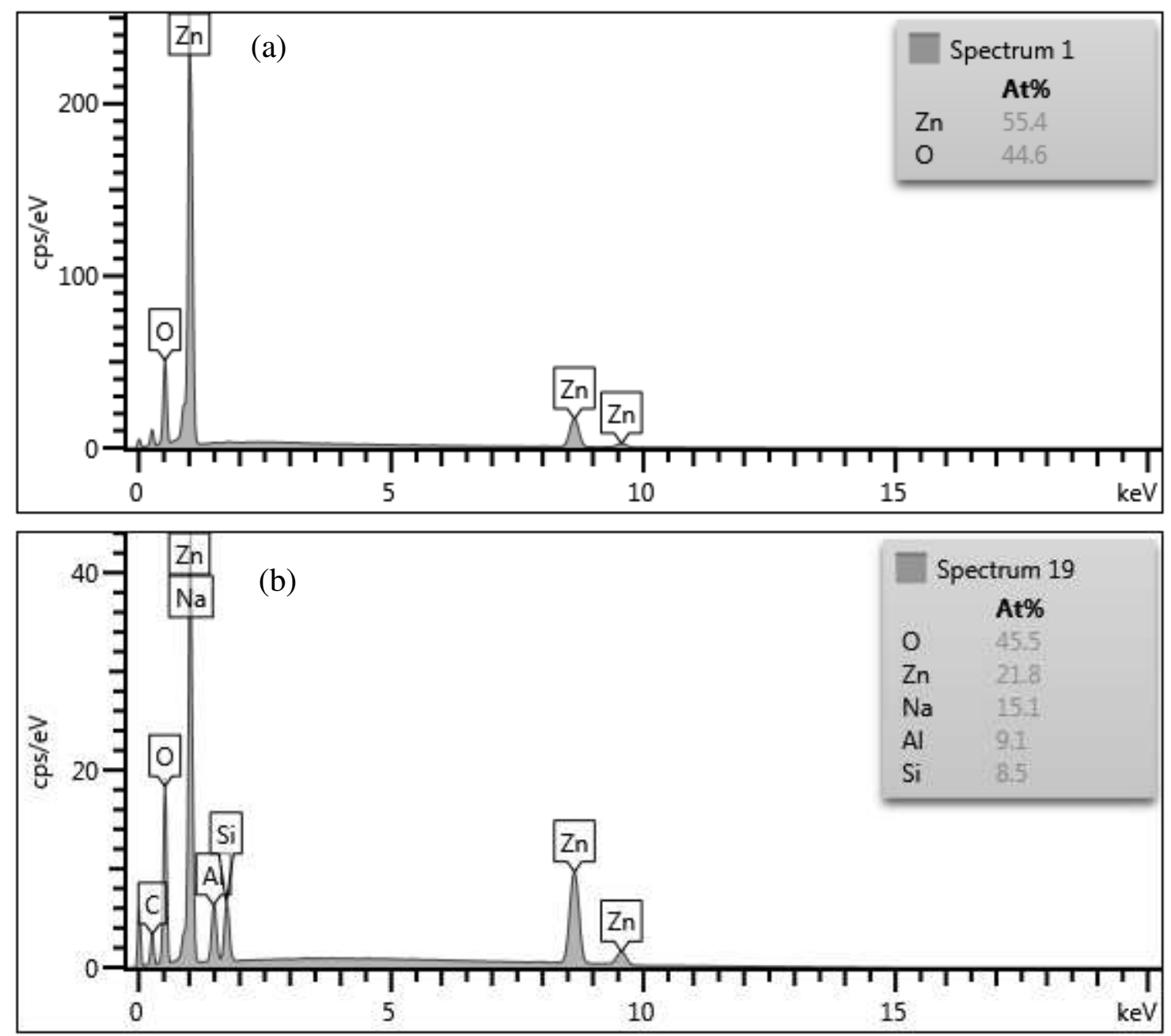

Fig. 4: EDX spectra for pure $\mathrm{ZnO}$ (a) and $\mathrm{ZnO} / \mathrm{Zeolite} \mathrm{Na}-\mathrm{A}$ (b)

\subsubsection{Microscopy images by TEM.}

Figure 5(a-f) shows transmission electron images (TEM) of pure zeolite $\mathrm{Na}-\mathrm{A}, \mathrm{ZnO}$ and the $\mathrm{ZnO} / \mathrm{Zeolite} \mathrm{Na}-\mathrm{A}$ catalyst. Zeolite revealed particle sizes of about $5 \mu \mathrm{m}$ whereas $\mathrm{ZnO}$ revealed particles hexagonal morphology of shapes ranging between 40-180 nm as indicated in figure 5(c). These images confirmed that the $\mathrm{ZnO} / \mathrm{Zeolite} \mathrm{Na}-\mathrm{A}$ composite catalyst was highly crystalline in nature. In addition the $\mathrm{ZnO}$ remain unchanged even after loading on to the zeolite surface. Figure 5(f) clearly shows $\mathrm{ZnO}$ particles attached on the zeolite surface which was in agreement with the results obtained by XRD and SEM. It was also seen that the $\mathrm{ZnO}$ nanoparticles on the zeolite surface were least agglomerated as compared to the pure ZnO. HRTEM shows particles of different morphology which could be mapped in to $\mathrm{ZnO}$ and zeolite. The Selected Area Electron Diffraction (SAED) shown in figure $5(\mathrm{~h})$ consist of concentric bright which implies that the $\mathrm{ZnO} /$ Zeolite Na-A is polycrystalline in nature. The obtained morphological study agrees with previous study by Sacco et al., [14]. 


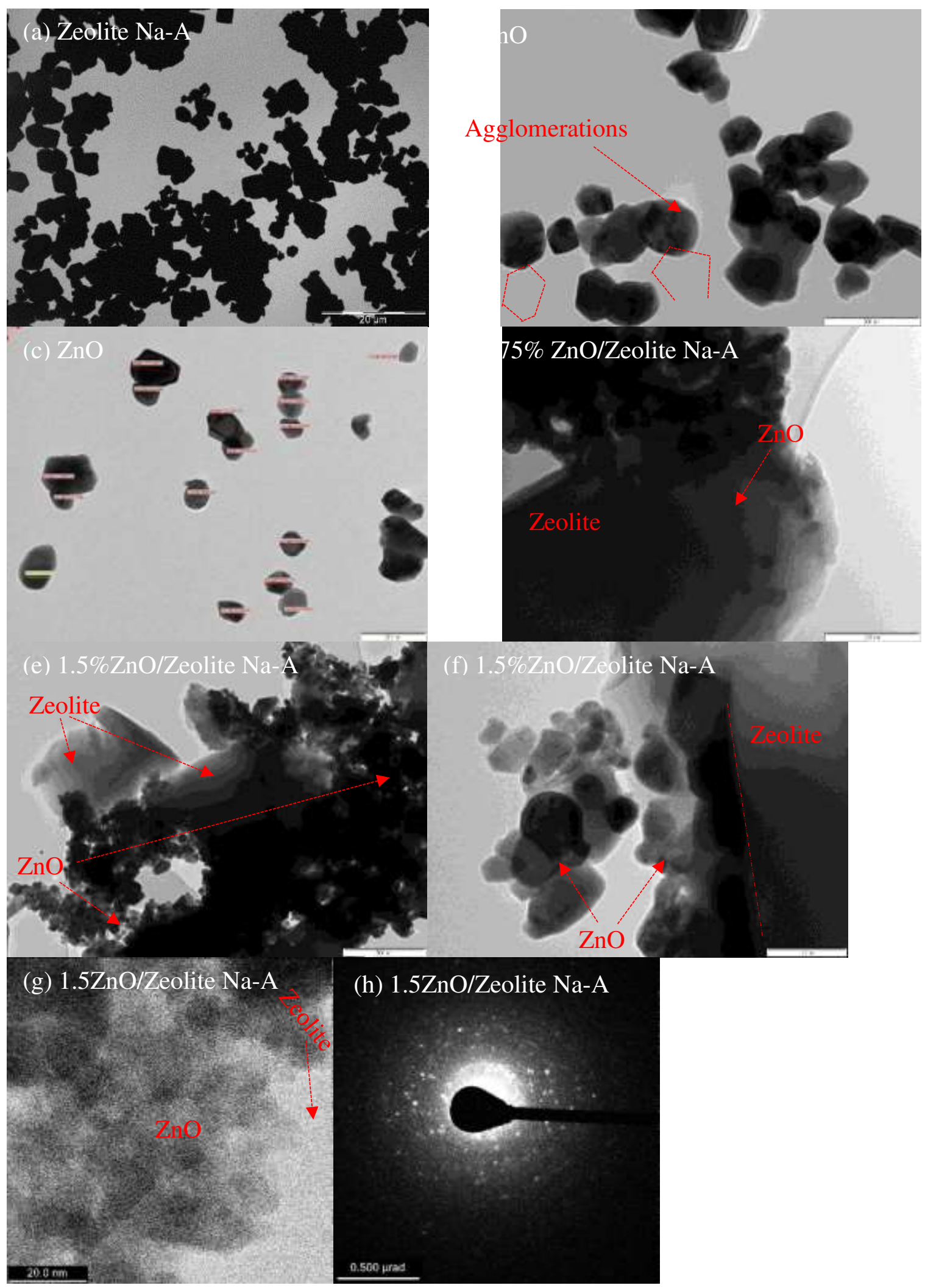

Fig. 5: Selected TEM images of zeolites, $\mathrm{ZnO}$ and $\mathrm{ZnO} /$ Zeolite Na-A are shown in 5 (a-f) whereas (g) and (h) shows HRTEM image and the Selected Area Electron Diffraction SAED respectively. 


\subsection{Nitrogen adsorption-desorption for surface area and pore size}

The adsorption behaviour of pure zeolite, pure $\mathrm{ZnO}$ and the $\mathrm{ZnO}$ /Zeolite Na-A NCs are shown in Fig. 6. All the three selected samples exhibited mesoporous type IV isotherm curve with hysteresis loop of type $\mathrm{H} 3$ according to the International Union of Pure and Applied Chemistry (IUPAC) classification. These types of isotherms are likely due characteristics of capillary condensation in parallel plate-shaped pores. Pure zeolites revealed the least amount of adsorbed $\mathrm{N}_{2}$ while the highest adsorption was observed on $\mathrm{ZnO} /$ Zeolite Na-A NC catalyst which implies an increase in adsorption properties when $\mathrm{ZnO}$ are loaded on the zeolites. This is caused by increased porosity due the presence of zeolitic micropores as well as mesopores formed by the aggregation of $\mathrm{ZnO}$ nanocrystallites on zeolite surface [15]. The Brunauer-Emmett-Teller (BET) surface area (S $\mathrm{S}_{\mathrm{BET}}$ ) for zeolite and $\mathrm{ZnO}$ was $0.40 \mathrm{~m}^{2} / \mathrm{g}$ and $3.32 \mathrm{~m}^{2} / \mathrm{g}$ respectively whereas for the selected sample of the $\mathrm{Zeo} / \mathrm{ZnO}$ catalyst was found to be $6.87 \mathrm{~m}^{2} / \mathrm{g}$. This increase in surface area of the prepared $\mathrm{ZnO} /$ Zeolite Na-A loaded catalyst indicated that the ZnO NPs may have formed a porous layer on the surface zeolite structure. Further, the formed, ZnO NPs layer, with a large surface area, provides a large number of functional groups that enhance the adsorption process [8]. The results show an increase of the surface area, a behaviour that is common when a replacement of a monovalent cation by a divalent one is carried out. When the $\mathrm{Zn}^{2+}$ exchange process occurs, one $\mathrm{Zn}^{2+}$ cation occupies the place of two $\mathrm{Na}^{+}$cations which leads to a surface area increment [10]. Increase in surface area of the catalyst leads to stronger adsorption properties thus enhanced photodegradation properties. On the other hand, the average pore size for $\mathrm{Zeo}, \mathrm{ZnO}$ and $\mathrm{ZnO} / \mathrm{Zeolite}$ Na-A were 81.12 $\AA$, $96.54 \AA$ and $149.70 \AA$ ̊ respectively.
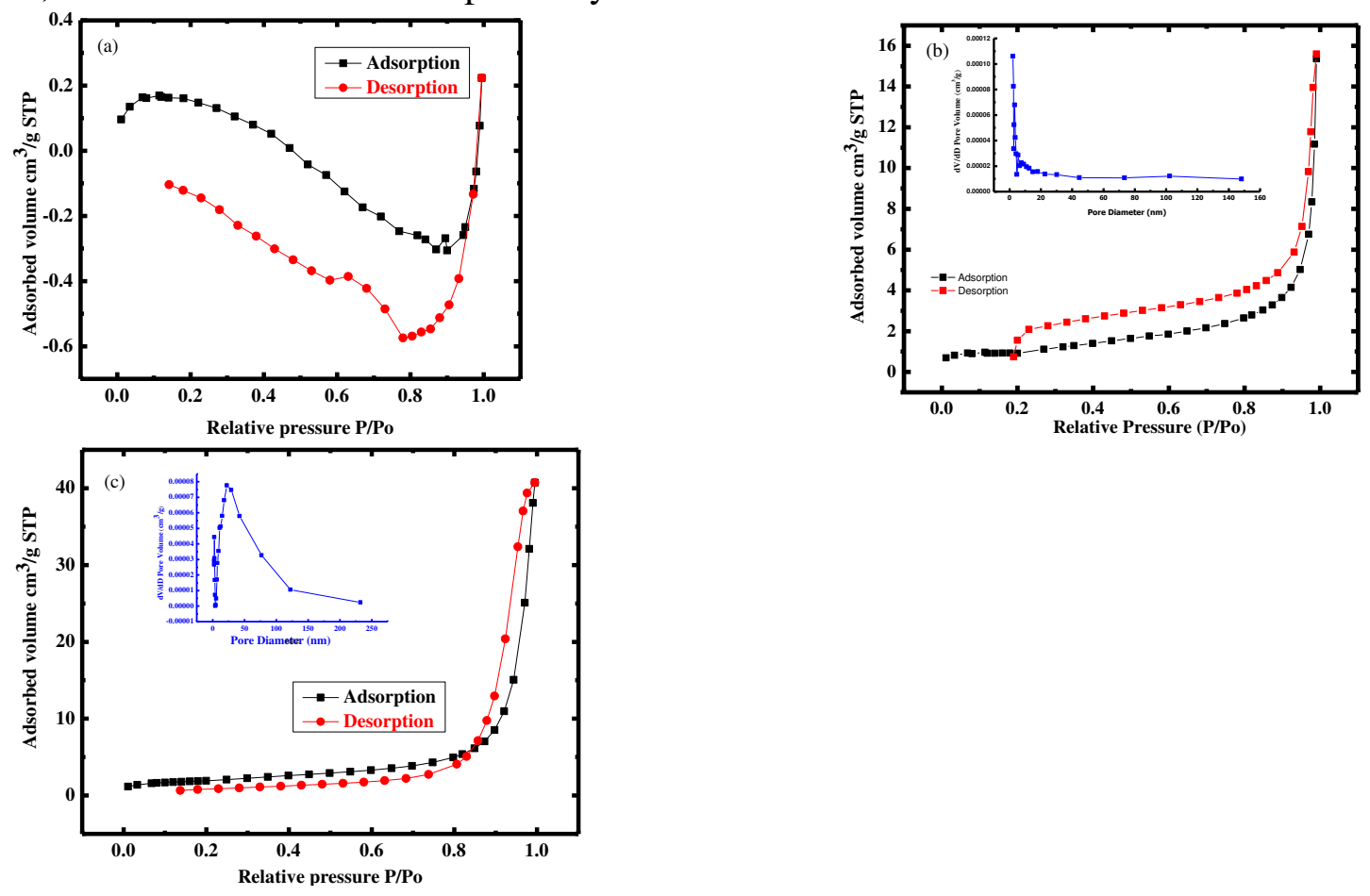

Fig. 6: Nitrogen adsorption-desorption curves for pure zeolite (a), pure $\mathrm{ZnO}$ (b) and $1.5 \% \mathrm{ZnO} / \mathrm{Zeolite} \mathrm{Na}-\mathrm{A}$ (c) nanopowders. Inset plots shows the respective pore size distribution. 
The Barrett-Joyner-Halenda $(\mathrm{BJH})$ pore size distribution curves are shown in the inset of fig. 6(b and c). The pore size distribution curve for $\mathrm{ZnO}$ shows a strong peak $2.38 \mathrm{~nm}$ and a weak one at about $7.78 \mathrm{~nm}$ while that of $\mathrm{ZnO} / \mathrm{Zeolite} \mathrm{Na}-\mathrm{A}$ sample revealed a broad peak centred at $22.6 \mathrm{~nm}$ clearly confirming that the majority of the pores were mesoporous. It was also observed that there was the presence of macro-pores which was as a result of spaces between $\mathrm{ZnO}$ on the surface of the zeolite or probably due to the zeolite structure itself. Generally it was observed that $\mathrm{ZnO}$ loaded catalyst had displayed higher porosity as compared to pure $\mathrm{ZnO}$ which leads to enhanced adsorption of the pollutant molecules and thus improving photocatalytic ability. The obtained textural properties of the Zno/Zeolite $\mathrm{Na}-\mathrm{A}$ catalyst were in agreement with results from previous studies such as Gayatri et al., [16] who worked on $\mathrm{ZnO} / Z$ eolite $\mathrm{Na}-\mathrm{A}$ and obtained $\mathrm{S}_{\mathrm{BET}}$, pore size and pore volume of $95.98 \mathrm{~m}^{2} / \mathrm{g}, 44.2 \AA$ and $0.08 \mathrm{~cm}^{3} / \mathrm{g}$ respectively. They attributed the obtained pore size to pore widening due to removal of impurities after the $\mathrm{ZnO} /$ zeolite activation treatment. A comparable results were also obtained by Mustapha et al., [17].

Table 2: Textural properties of zeolite, $\mathrm{ZnO}$ and $\mathrm{ZnO} / \mathrm{Zeolite} \mathrm{Na}-\mathrm{A}$ composite.

\begin{tabular}{llll}
\hline Sample & $\begin{array}{l}\text { BET surface area } \\
\mathrm{S}_{\mathrm{BET}}\left(\mathrm{m}^{2} / \mathrm{g}\right)\end{array}$ & $\begin{array}{l}\text { Pore size } \\
(\AA)\end{array}$ & $\begin{array}{l}\text { Pore volume } \\
\left(\mathrm{cm}^{3} / \mathrm{g}\right)\end{array}$ \\
\hline \hline Zeolite & 0.40 & 81.12 & 0.000405 \\
$\mathrm{ZnO}$ & 3.32 & 96.54 & 0.008002 \\
$\mathrm{Zeo} / \mathrm{ZnO}$ & 91.73 & 149.70 & 0.000466 \\
\hline
\end{tabular}

\subsection{Optical properties}

\subsubsection{UV-Vis DRS spectroscopy}

The optical properties of zeo, $\mathrm{ZnO}$ and $\mathrm{ZnO} / \mathrm{Zeolite} \mathrm{Na}-\mathrm{A}$ with various $\mathrm{ZnO}$ loads were obtained by running the UV-visible diffuse reflectance spectroscopy on the samples in the wavelength range $200 \mathrm{~nm}-800 \mathrm{~nm}$. The reflectance of pure zeolite was high in the visible light region with a gentle decline in the UV implying slight absorption in the UV region. A peak in this region at around $254 \mathrm{~nm}$ which is a characteristic absorbance peak associated with zeolite was also observed [18]. These absorption bands in the short-wavelengths are inherent in different types of zeolites and they are likely to have originated from the charge transfer $\mathrm{O}_{2}^{-} \rightarrow \mathrm{Al}^{3+}$ which involve the participation of aluminum atoms at specific locations such as surface, corners, defects, etc [13]. The $\mathrm{ZnO}$ and zeo/ $\mathrm{ZnO}$ samples exhibited reflectance spectrum quite different from that of pure zeolite powder. A sharp rise in reflectance around wavelength $\lambda=368 \mathrm{~nm}$ for the pure $\mathrm{ZnO}$ powder is due fundamental absorption from valence band to the conduction band due to the $O^{2-} \rightarrow$ $\mathrm{Zn}^{2+}$ ligand to metal charge transfer transition [19]. Moreover, the spectra of $\mathrm{ZnO} / \mathrm{Zeolite}$ $\mathrm{Na}-\mathrm{A}$ was different to that of pure zeolite sample which was due to the $\mathrm{ZnO}$ loading. On the contrary $\mathrm{ZnO} / \mathrm{Zeolite} \mathrm{Na}-\mathrm{A}$ showed higher reflectance intensity in the visible region attributed to the $\mathrm{ZnO}$ particles embedded on zeolite. A slight wavelength shift associated with $\mathrm{ZnO}$ nanoparticle loading on the zeolite surface was also observed between $359 \mathrm{~nm}$ and $387 \mathrm{~nm}$ which could be caused by variation in crystallite sizes. 

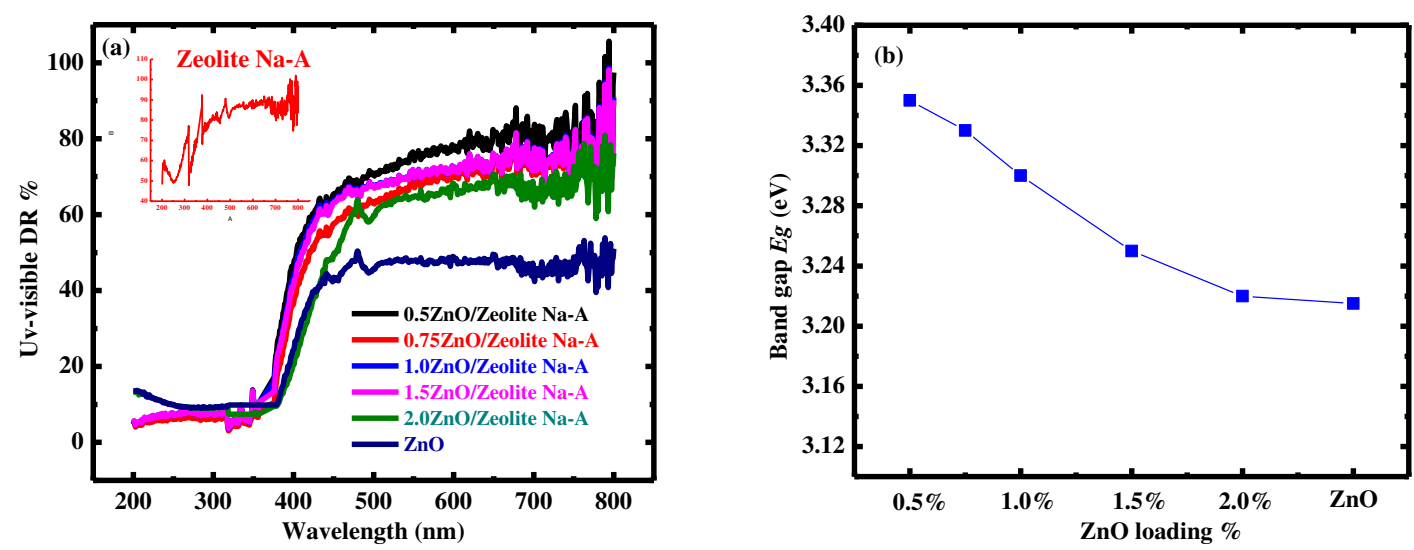

Fig. 7: Diffuse reflectance spectroscopy (DRS) of pure Zeolite, pure $\mathrm{ZnO}$ and the $\mathrm{ZnO} /$ Zeolite Na-A loaded catalyst.

\subsubsection{Optical band gap determination}

In order to calculate the band gap $E_{g}$, reflectance $R$ is converted to the equivalent absorption coefficient using the modified Kubelka-Munk function given by Eq. 7;

$$
F(R)=\frac{(1-R)^{2}}{2 R}
$$

Where $F(R)$ (Kubelka Munk function) is proportional to the absorption coefficient $\alpha$ and $R$ is the reflectance.

Given that;

$$
\alpha=\frac{4 \pi k}{\lambda}
$$

Here, $\lambda$ is the wavelength of the absorbed photon and $k$ is the extinction coefficient. The bandgap of the composites can easily be obtained extrapolating the linear portion specifically where the absorption begins of the plot of $(\alpha h v)^{\frac{1}{n}}$ versus $h v$ to the $h v$ axis of the Tauc equation;

$$
F(R) \times h v=A\left(h v-E_{g}\right)^{n}
$$

The proportionality constant is denoted by $A$ and is related to the nature of the material, $h$ is Planck's constant $\left(6.63 \times 10^{-34} \mathrm{JS}^{-1}\right)$, whereas $n=0.5$ for direct allowed transitions and $n=2$ for indirect transitions respectively. Since $\mathrm{ZnO}$ is a direct band gap semiconductor $n=0.5$ was applied in evaluating the band gap $E_{g}$.

The variation of obtained band gap $E_{g}$ with zeolite addition shown in figure 7 (b) revealed the band gap for $\mathrm{ZnO}$ loaded catalyst was larger than that of pure $\mathrm{ZnO}$ due the nonconducting nature of zeolites. As the amount of $\mathrm{ZnO}$ increased, the band gap approached the band gap value of pure $\mathrm{ZnO}$. The reason for the decrease in bandgap $E g$ is due increase in crystallite sizes. A band gap decline is useful in increased wavelength response necessary for the catalytic photodegradation. 


\subsection{Functional groups by FTIR}

The FTIR spectra for the determination of the functional groups in zeolite, $\mathrm{ZnO}$ and Zeolite $\mathrm{Na}-\mathrm{A}$ were obtained in the wavelength range $400-4000 \mathrm{~cm}^{-1}$ as shown in figure 8 . The broad band in the region $3607-3200 \mathrm{~cm}^{-1}$ and $1657 \mathrm{~cm}^{-1}$ in the zeolite spectrum is assigned to the vibration stretching of $\mathrm{O}-\mathrm{H}$ due the zeolitic water $\left(\mathrm{H}_{2} \mathrm{O}\right)$ entrapped in the micro porous structure of the zeolite frame work [11]. The FTIR peaks between $1200 \mathrm{~cm}^{-1}$ and $400 \mathrm{~cm}^{-1}$ are assigned to $\mathrm{Si}-\mathrm{O}-\mathrm{Al}$ and $\mathrm{Si}-\mathrm{O}$ bending vibration associated with the tetrahedral or alumina- and silico-oxygen bridges of the zeolite aluminosilicate internal structures [13]. Pure $\mathrm{ZnO}$ revealed weak peaks $\mathrm{O}-\mathrm{H}$ in a similar region to the of Zeolites Na-A $\left(3426 \mathrm{~cm}^{-}\right.$ ${ }^{1}$ ) and $1633 \mathrm{~cm}^{-1}$ due to absorbed atmospheric water vapour [20,21]. The weak peaks on the sample located at $1379 \mathrm{~cm}^{-1}$ are caused by $-\mathrm{CH}_{3}$ sourced from titanium tetraisopropoxide and 2-propanol (precursors) [22] whereas the peak at $877 \mathrm{~cm}^{-1}$ is assigned to $\mathrm{Zn}-\mathrm{O}$ stretching. This precursor peak was missing in pure zeolite sample. $\mathrm{Zn}-\mathrm{O}$ characteristic absorption peaks are located around $450 \mathrm{~cm}^{-1}$ (not visible) [23]. All $\mathrm{ZnO} / \mathrm{Zeolite} \mathrm{Na}-\mathrm{A}$ samples revealed peaks in similar positions to those of zeolites. This is because there was no peak shift due the zeolite treatment and $\mathrm{ZnO}$ loading since zeolite structure does not change when a transition metal oxide cation leaks in to the zeolite structure [24]. This indicates high stability of the $\mathrm{ZnO}$ /zeolite which is in agreement with earlier XRD and SEM results. There were faint bands associated with Si-O-Zn or Zn-O-Al detected about $668 \mathrm{~cm}^{-1}$ which implies that the interaction of zeolites with $\mathrm{ZnO}$ may likely have occur only on surface of the zeolites. However the broadening of the $\mathrm{ZnO}$ peak at $1032 \mathrm{~cm}^{-1}$ assigned to $\mathrm{Zn}-\mathrm{O}-\mathrm{ZnO}$ stretching may be due to the combination of $\mathrm{ZnO}$ band and the original vibrational bands of the zeolite framework [25]. The FTIR study shows that the $\mathrm{ZnO} /$ zeolite $\mathrm{Na}-\mathrm{A}$ formed strong bonds between the $\mathrm{ZnO}$ and the zeolite surface which ensures that the $\mathrm{ZnO}$ nanoparticles strongly adhered to the zeolite surface throughout the photodegradation and recycling process.

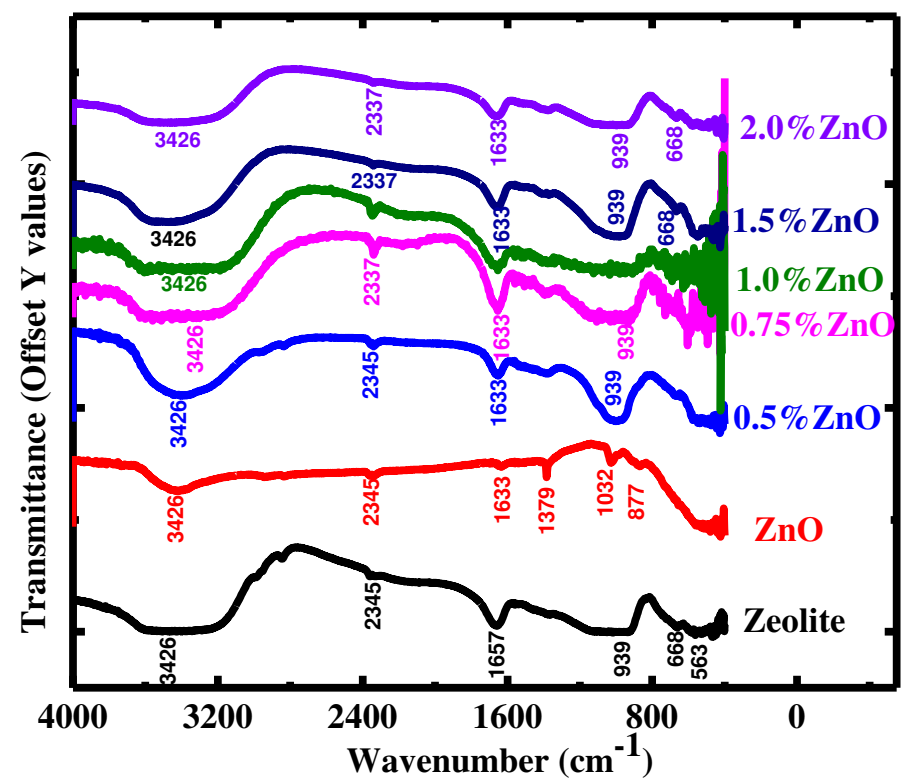

Fig. 8: FTIR spectra of indicating various functional groups on Zeolite, pure $\mathrm{ZnO}$ and $\mathrm{ZnO} /$ Zeolite Na-A 


\subsection{Photodegradation measurements}

\subsubsection{Photocatalytic dye removal.}

To test the effects of $\mathrm{ZnO}$ loading on the photodegradation a study was conducted on its ability to photodegrade $\mathrm{MB}$ in water as the model pollutant. The photocatalytic experiments were conducted as per the procedure explained in section 2.3.1 above and the obtained results were as shown in figure 9. From the figure it is clear that there was minimum degradation of the dye on UV but without the catalyst (photolysis). Similar curves were also obtained from the adsorption studies by the $\mathrm{ZnO} /$ Zeolite Na-A catalyst. When the degradation experiments were conducted in both the presence of the UV and the catalyst as shown in figure $9 \mathrm{~b}$ the dye was easily degraded leading to almost the disappearance of the MB main peaks at $662 \mathrm{~nm}$ in $360 \mathrm{~min}$. This implies that the decrease in the peaks intensity was proportional to the loss in the Uv-visible absorbance ability and was caused by the photodegradation effects of the catalyst. The intensity of the MB peaks is the measure of the dyes concentration from which we can conclude that the decrease in peaks is associated with the breakdown of the MB internal structures.
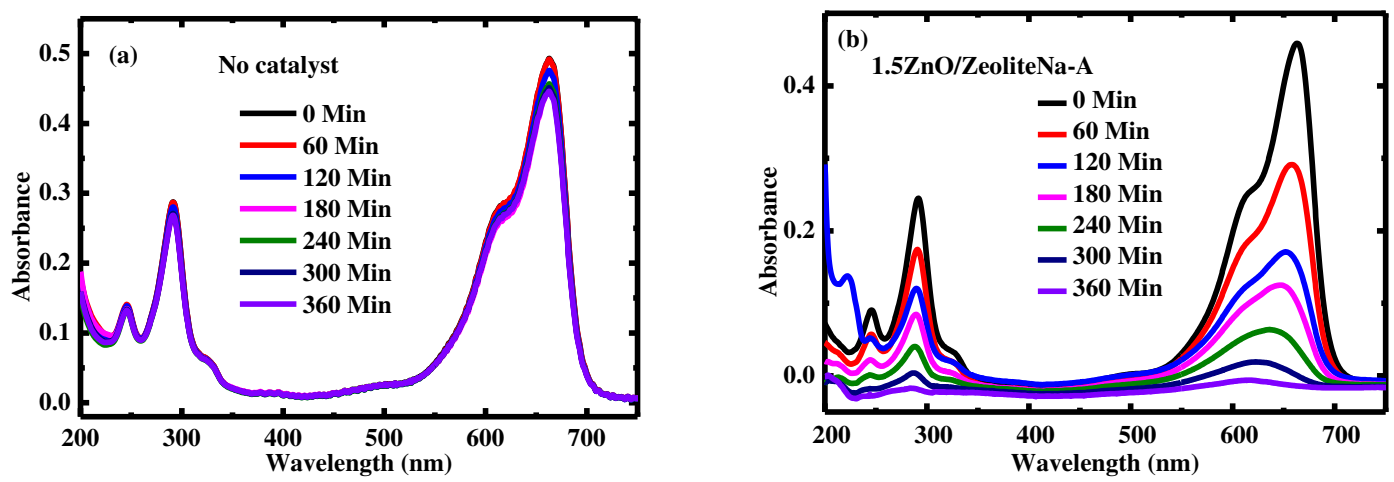

Fig. 9: Effects of change of methylene blue dye peak with time on exposure to UV radiation (a) blank (b) with catalyst

\subsubsection{Kinetics of photodegradation}

To study the degradation kinetics, it was assumed that the photodegradation process of the methylene blue dye assumes pseudo first-order kinetics. This was achieved via the plots of the best fit of $\ln C / C_{o}$ versus time in minutes (Fig. 10) where by their gradients were evaluated in order to determine the rates constant $k$ and the statistical correlation coefficient $R^{2}$ values. The obtained values are as shown in Table 3 .

From the plots, $\mathrm{ZnO} / \mathrm{Zeolite} \mathrm{Na}-\mathrm{A}$ revealed higher photodegradation rates compared to pure $\mathrm{ZnO}$ nanopowders which is probably caused by the combine surface effects of $\mathrm{ZnO}$ on the zeolite surface such as high electron mobility and suppressed electron hole recombination. There was an increase of photodegradation rate with increase in the $\mathrm{ZnO}$ loading with $1.5 \% \mathrm{ZnO} / \mathrm{Zeolite} \mathrm{Na}-\mathrm{A}$ showing the optimum rate of photodegradation from which a decline was observed. The reason for the high rate could be due to the synergetic effect of loading in conjunction with optimum dispersion of $\mathrm{ZnO}$ nanoparticles. Such a case, ensures availability of exposed active sites when $\mathrm{ZnO}$ is loaded on to the zeolites. On the other hand the accessible porous zeolite can absorb the MB and increase the contact time with catalyst [26]. It was noted that excessive loading led to decline of 
photodegradation rate for obvious reason that agglomerations would contribute to effective decrease in the surface area.
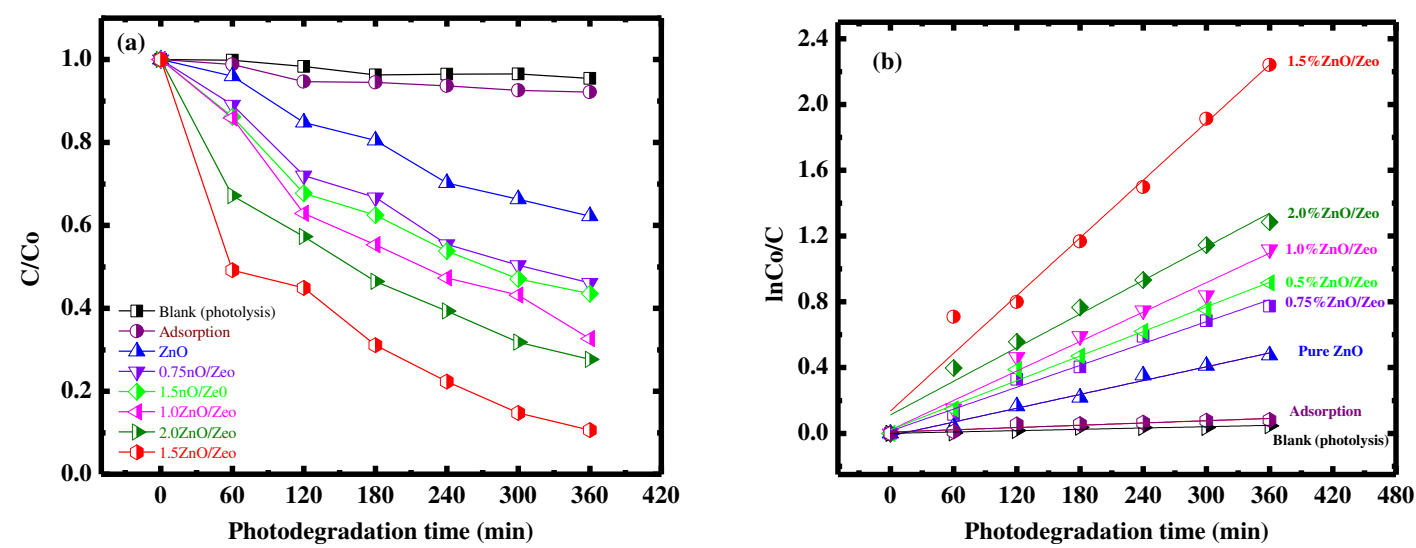

Fig. 10: (a) $\mathrm{C} / \mathrm{Co}$ versus degradation time (b) the various degradation rates for different $\mathrm{ZnO}$ loading levels.

Table 3: The calculated rates $\mathrm{k}$ of photodegradation and $\mathrm{R}$ square for different $\mathrm{ZnO}$ loading.

\begin{tabular}{lcl}
\hline Sample name & $\begin{array}{l}\ln \left(C_{o} / C\right)=k t \\
k \text { value } \times 10^{-3}\left(\mathrm{~min}^{-1}\right)\end{array}$ & $\begin{array}{l}\text { Regression } \\
\text { square } \mathrm{R}^{2}\end{array}$ \\
\hline \hline Blank (Photolysis) & 1.36 & 0.8518 \\
Adsorption & 2.17 & 0.8677 \\
Bare ZnO & 1.41 & 0.9830 \\
$0.5 \% \mathrm{ZnO} / Z$ Zeolite Na-A & 2.52 & 0.9887 \\
$0.75 \% \mathrm{ZnO} / Z$ Zeolite Na-A & 2.22 & 0.9832 \\
$1.0 \% \mathrm{ZnO} /$ Zeolite $\mathrm{Na}-\mathrm{A}$ & 2.98 & 0.9770 \\
$1.5 \% \mathrm{ZnO} / Z$ Zeolite $\mathrm{Na}-\mathrm{A}$ & 5.79 & 0.9756 \\
$2.0 \% \mathrm{ZnO} /$ Zeolite $\mathrm{Na}-\mathrm{A}$ & 3.40 & 0.9748 \\
\hline
\end{tabular}

\subsubsection{The proposed mechanism of degradation}

The pure $\mathrm{ZnO}$ structure has $\mathrm{Zn}-\mathrm{O}$ bonds whereas new bonds such as $\mathrm{Zn}-\mathrm{O}-\mathrm{Al}$ and $\mathrm{Zn}-\mathrm{O}-$ $\mathrm{Si}$ may be formed when $\mathrm{ZnO}$ was loaded on the zeolites surface. This results in traces $\mathrm{ZnO}$ being incorporated in to aluminosilicate framework of zeolite which has a high thermal and chemical stability [25]. In the proposed mechanism of photodegradation, $\mathrm{ZnO}$ on absorbs UV radiation whereby electrons are excited from the valence band to conduction band simultaneously generating holes at the valence band (Fig.11). The generated electrons interact with atmospheric oxygen producing superoxide radicals whereas the holes interact with water to produce $\mathrm{OH}$ radicals. These active radicals interact with the adsorbed dye molecules causing destruction of the dye molecular structure. However for the pure $\mathrm{ZnO}$ this process is highly hindered by charge recombination as the excited electron fall back and recombine with holes.

When $\mathrm{ZnO}$ semiconductor is loaded on the zeolites, the entire process is altered; the $\mathrm{ZnO}$ absorbs the UV radiation generating electron-hole pairs. The electrons diffuse in to the 
aluminosilicate framework of the zeolites thus getting trapped [27]. In such situations, the charge recombination process is highly slowed down extending the reaction time with dye molecules. In addition, the generated hydroxyl ions and super radicals are readily transferred from the $\mathrm{ZnO}$ to the zeolite surface where organic pollutant molecules are already adsorbed maintaining prolonged contact between the pollutant and radicals [28].

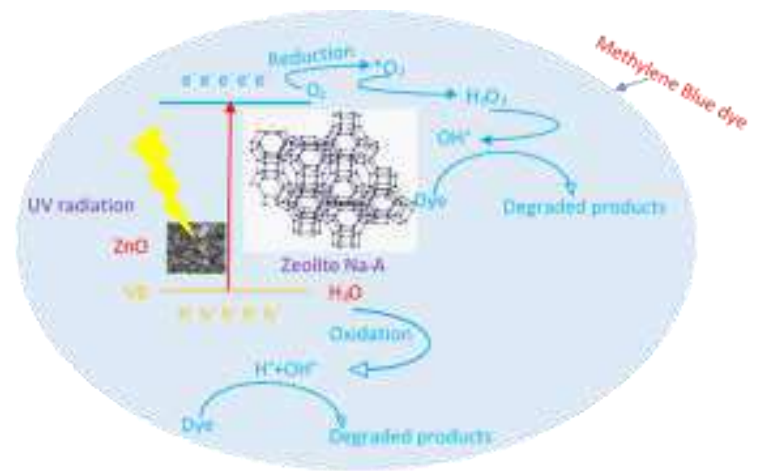

Figure 11: Photodegradation mechanism

\section{Mineralization}

Discoloration rate of MB dye is an indication of disintegration of the basic structures of the dye which is reflected in the decrease in intensity of the dyes main peaks. To ascertain if the dye underwent complete mineralization the amount of organic matter in the dye after photodegradation was determined after conducting the chemical oxygen demand (COD) experiments. Table 4 shows the percentage of decrease in COD for the respective catalyst samples. From the table it can be seen that after 6 hours of continuous irradiation, there was remarkable reduction of the percentage of COD recorded implying disintegration of MB's organic matter for most samples. However, the blank sample COD reduction was insignificant indicating that associating the reduction on the catalysts effect. A reduction in COD reflects the extend of photodegradation and mineralization of organic pollutants. The decrease in the level of COD reflected a trend similar to the obtained photocatalytic discoloration of the main absorption peak intensity of the methylene blue dye.

Table 4: The various decrease in $\mathrm{COD}$ of the $\mathrm{ZnO} / \mathrm{Zeolite} \mathrm{Na}-\mathrm{A}$ catalyst

\begin{tabular}{lllllllll}
\hline \multicolumn{7}{l}{ Percentage (\%) COD } \\
$\begin{array}{l}\text { Time } \\
\text { (min) }\end{array}$ & Blank & Zeo Na-A & ZnO & $0.5 \% \mathrm{ZnO}$ & $0.75 \% \mathrm{ZnO}$ & $1.0 \% \mathrm{ZnO}$ & $1.5 \% \mathrm{ZnO}$ & $2.0 \% \mathrm{ZnO}$ \\
\hline 0 & 100 & 100 & 100 & 100 & 100 & 100 & 100 & 100 \\
60 & 99.7 & 86.1 & 77.8 & 52.6 & 74.9 & 64.8 & 82.7 & 75.6 \\
120 & 99.1 & 62.6 & 48.9 & 26.7 & 45.9 & 40.4 & 52.8 & 47.0 \\
180 & 98.7 & 47.1 & 30.5 & 18.9 & 28.8 & 27.9 & 33.9 & 30.1 \\
240 & 98.1 & 39.7 & 26.3 & 14.4 & 22.2 & 16.5 & 32.7 & 23.2 \\
300 & 97.9 & 35.1 & 24.7 & 10.6 & 20.3 & 12.7 & 29.1 & 22.9 \\
360 & 97.4 & 34.5 & 23.9 & 9.6 & 16.7 & 10.5 & 25.5 & 21.4 \\
\end{tabular}




\subsubsection{Role of the zeolite framework}

After the experiments, ZnO/Zeolite Na-A revealed higher photocatalytic activity compared to pure Zeo or ZnO. Zeolite are photocatalytically inactive, are suitable for use as support materials, whereas in unsupported $\mathrm{ZnO}$ low catalytic efficiency is associated with particle agglomerations as noted in SEM and TEM images. $\mathrm{ZnO}$ nanoparticles have high tendencies of agglomeration to due to large surface energies which is usually weakened by loading it on the zeolite [29]. The reason behind high photocatalytic activity in $\mathrm{ZnO} / \mathrm{ZeoliteNa}-\mathrm{A}$ is attributed to well dispersed $\mathrm{ZnO}$ nanoparticles on the zeolite support frame work which ensures high surface area. $\mathrm{ZnO}$ nanoparticles are embedded on zeolite active site where the exchange active ions with the zeolites. During the exchange, electrons from $\mathrm{ZnO}$ Evв are excited to the $\mathrm{E}_{\mathrm{CB}}$ of self then diffuse into oxygen $(\mathrm{O})$ atoms and finally to the aluminium atoms in the zeolite framework [30] hence preventing electron/hole recombination which causes increased photodegradation efficiency. Besides, zeolites have high adsorption capacity of the dye molecules which bring them close to the surface of catalyst molecules increasing their chances of being attacked by the $\mathrm{OH}$ radicals. These $\mathrm{OH}$ radicals are also trapped in the frame work of the zeolite where the dye molecules are adsorbed enhancing further degradations reactions. It is also believed that zeolite framework can also participate actively in the catalytic process through hindering charge recombination by acting either as an electron donor or acceptor. This means that it is likely that some $\mathrm{Zn}^{2+}$ cations in $\mathrm{ZnO} /$ zeolite Na-A may bind to the zeolite and interact with its anionic $\left(\mathrm{AlO}_{2}^{-}\right)$framework triggering anion-cation pairs which results in strong electrostatic properties leading to increase in adsorption of the cationic MB dye hence the increases the degradation extent of the pollutant [31]. These results demonstrate that the active centers are composed of the $\mathrm{ZnO}$ on the zeolite structure.

\subsubsection{Effects of scavengers and $\mathrm{H}_{2} \mathrm{O}_{2}$ on the active radicals}

Further investigation was performed to determine the nature of primary active species in the photocatalytic process. To verify if holes, electrons or hydroxyl radical are involved in the photocatalytic degradation of $\mathrm{MB}$ on the pure $\mathrm{ZnO}$, zeolite and zeolite supported $\mathrm{ZnO}$ nanoparticles, the photocatalytic activity was carried out in the presence of different scavengers. The procedure for photodegradation was repeated as described in section 2.3.4 above using potassium iodide, isopropanol and ascorbic acid as hole $(h+)$, hydroxide radical $\left(\mathrm{OH}^{*}\right)$ and superoxide $\mathrm{O}_{2}^{--}$radical scavengers respectively [32]. The rate of degradation of $\mathrm{MB}$ over zeolite, $\mathrm{ZnO}$ and $\mathrm{ZnO} /$ Zeolite $\mathrm{Na}-\mathrm{A}$ supported $\mathrm{ZnO}$ follows the order of $\mathrm{OH}^{*}>$ holes $\mathrm{h}^{+}>$electrons which shows that $\mathrm{OH}^{*}$ radicals played the important role on MB degradation rate (Fig. 12). The possible reaction equations are shown;

$$
\text { Catalyst }+h v \rightarrow \text { Catalyst }+e_{C B}^{-}+h_{V B}^{+}
$$

Charge carrier recombination may occur after charge separation resulting to heat

$$
e_{C B}^{-}+h_{V B}^{+} \rightarrow \text { heat }
$$

Without recombination, holes in the valence band $h_{V B}^{+}$attack adsorbed water as shown in equation 


$$
\begin{aligned}
& \mathrm{H}_{2} \mathrm{O}_{a d s}+h_{V B}^{+} \rightarrow \mathrm{H}^{+}+O H_{a d s}^{*} \\
& O H_{a d s}^{-}+h_{V B}^{+} \rightarrow O H_{a d s}^{*}
\end{aligned}
$$

On the other hand electrons in the conduction band $\left(e_{C B}^{-}\right)$attack adsorbed atmospheric oxygen resulting to production of the superoxide radical $\left(\mathrm{O}_{2}^{*-}\right)$.

$$
\begin{gathered}
\left(\mathrm{O}_{2}\right)_{a d s}+e_{\mathrm{CB}}^{-} \rightarrow \mathrm{O}_{2}^{*-} \\
\mathrm{O}_{2}^{*-}+\mathrm{H}^{+} \rightarrow \mathrm{HO}_{2}^{*} \\
2 \mathrm{HO}_{2}^{*} \rightarrow \mathrm{H}_{2} \mathrm{O}_{2}+\mathrm{O}_{2} \\
\mathrm{H}_{2} \mathrm{O}_{2}+e_{\mathrm{CB}}^{-} \rightarrow \mathrm{OH}^{*}+\mathrm{OH}^{-} \\
\mathrm{MB}+\mathrm{OH}_{\text {ads }}^{*} \rightarrow \text { Ads Radicals }+\mathrm{H}_{2} \mathrm{O} \rightarrow \text { degradation products }
\end{gathered}
$$

$M B+h_{V B}^{+} \rightarrow$ Ads Radicals $\rightarrow$ degradation products

However, with regard to photocatalytic degradation of $\mathrm{MB}$ upon addition of oxidants, it was found that the adding of hydrogen peroxide $\left(\mathrm{H}_{2} \mathrm{O}_{2}\right)$, promoted the photodegradation rate. Increase in the photodegradation rate of $\mathrm{MB}$ dye is attributed to the reaction between electrons on the conduction band of $\mathrm{ZnO}$ in the catalyst with hydrogen peroxide as in equation. Hydrogen peroxide is a better electron acceptor as compared to the dissolved oxygen and thus can act as an alternative electron acceptor which hinders electron-hole recombination as per equation

$$
\begin{aligned}
& \mathrm{H}_{2} \mathrm{O}_{2}+e_{C B}^{-} \rightarrow \mathrm{HO}^{-}+\mathrm{HO}^{*} \\
& \mathrm{H}_{2} \mathrm{O}_{2}+h v \rightarrow 2 \mathrm{HO}^{*}
\end{aligned}
$$

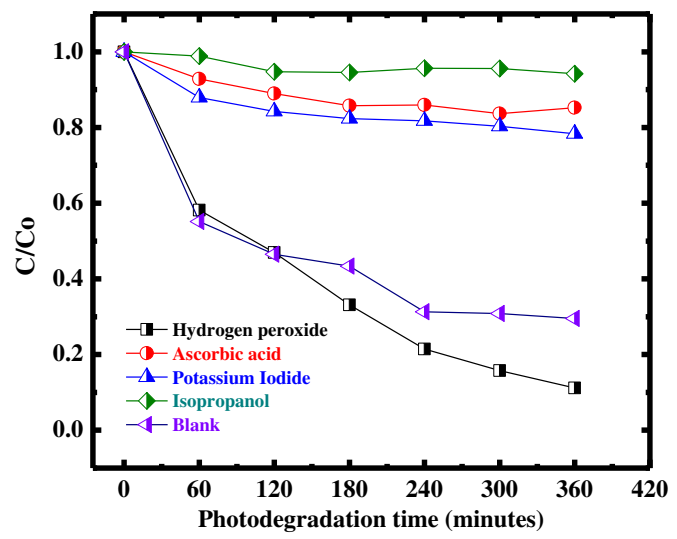

Fig. 12: Effect of different radical scavengers and oxidant 


\subsubsection{Effect of catalyst dosage}

To determine the optimum amount of catalyst dosage required for a particular application, the photodegradation was repeated with application of different amounts keeping the other factors constant. The catalyst dosage applied ranged $0.1 \mathrm{~g}$ to $1.0 \mathrm{~g} / \mathrm{L}$ for each of the experiments and various degradation the rates $k$ obtained by plotting $\ln \mathrm{C} / \mathrm{Co}$ verses time (min). To obtain the relationship between the catalyst loadings, the various degradation constants obtained were plotted against the mass of the catalyst dosage (mg). From the results obtained in figure 13, the degradation constant $\mathrm{k}$ increased with increase in the catalyst dosage. When the amount of catalyst was increased from $0.03 \mathrm{~g}$, the degradation constant $k$ increased from 5.79 to $7.49 \mathrm{~min}^{-1}$ beyond which a decline in $\mathrm{k}$ value was observed. This implies the optimum catalyst dosage lies around $0.12 \mathrm{~g}$ although it advisable to use a slightly lower amount which will gives almost similar results at lower cost. The main reasons for declined performance beyond $0.12 \mathrm{~g}$ include the aggregation of the catalyst reducing the exposed surface area with active sites. Further too much catalyst may mask, reflect or scatter light thus preventing its penetration which reduces photon absorption charge separation thus reducing the photodegradation efficiency. In addition aggregation may result in to deactivation of the catalyst due to collisions between the excited $\mathrm{ZnO}$ and ground state $\mathrm{ZnO}$ atoms when in the same vicinity and therefore the right amount should be added to avoid excesses.

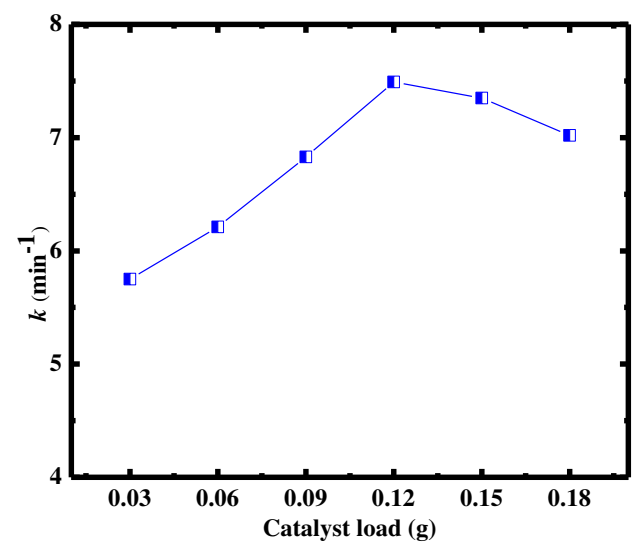

Fig. 13: Variation between degradation constant $k$ against $\mathrm{ZnO} /$ Zeolite Na-A catalyst loading

\subsubsection{Effect of initial MB concentration}

The initial concentration of MB dye may affect the photodegradation efficiency of the catalyst. In order to determine the effect of the substrates initial concentration on the rate of photodegradation, several experiments were conducted with varying $\mathrm{MB}$ concentrations ranging $20 \mathrm{mmol} / \mathrm{L}$ to $70 \mathrm{mmol} / \mathrm{L}$ on the optimum catalyst loading of $1.5 \mathrm{ZnO} / \mathrm{zeolite} \mathrm{Na-}$ A sample. The rate of photodegradation increased with increase in initial concentration of $\mathrm{MB}$ up to $5 \mathrm{ommol} / \mathrm{L}$ after which a decline in the rate was observed as in figure 14 . Usually the generated active radicals such as hydroxyl ions have limited lifetime in the nanosecond range and in most cases their action is localized around where they are formed. This implies that when the MB concentration is increased, the number of dye molecules per unit volume is also increased which in turn increases the chances of these dye molecules reacting with hydroxyl ions. However as the MB concentration exceeds a certain limit, excess adsorption of the dye on the catalysts occurs thus blocking the active sites. Excess dye result to high 
adsorption of the dye which may block the incoming light photons from reaching the catalyst limiting the catalyst's absorption and excitation. These results in to a decrease in charge carrier separation. Simultaneously, a large amount of intermediates and other organic substances may probably adsorbed on the surface of the catalyst, making it more difficult for holes to enter the solution contact to the catalyst surface. Table 5 shows the various photodegradation constants for various MB concentrations.
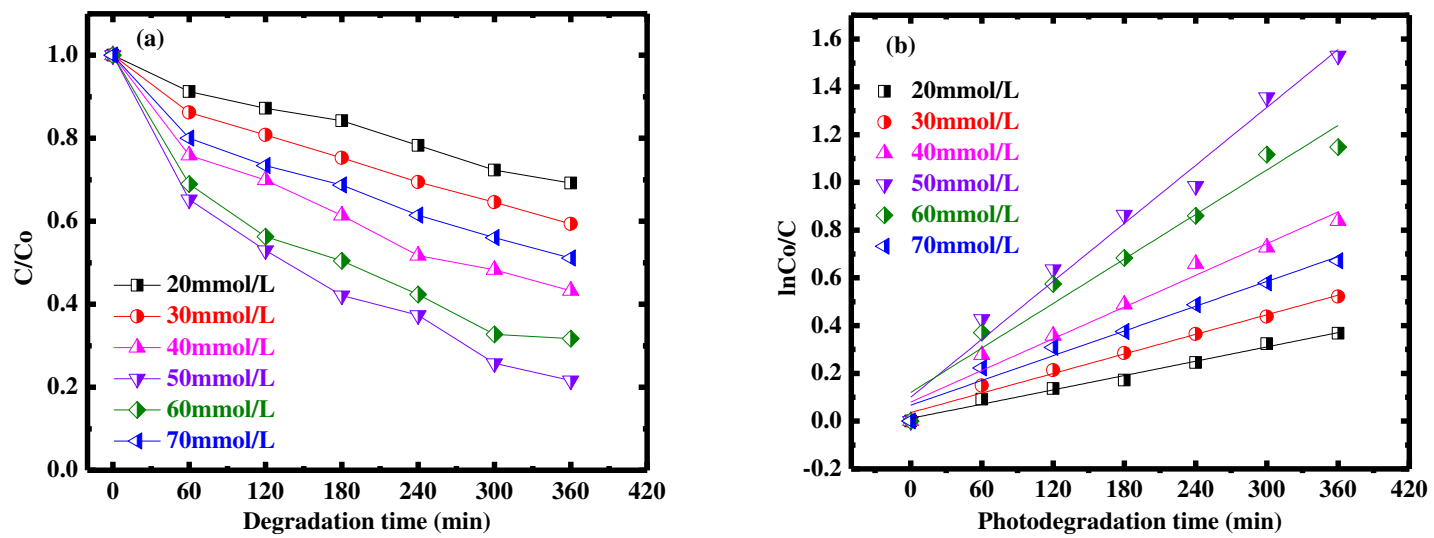

Fig. 14: (a) C/Co versus degradation time at differenet MB concentarations (b) the various degradation rates for at different $\mathrm{MB}$ concentrations.

Table 5: The calculated rates $\mathrm{k}$ of photodegradation and $\mathrm{R}$ square for different MB concentrations

\begin{tabular}{lll}
\hline MB concentration & $\begin{array}{l}\ln \left(C_{o} / C\right)=k t \\
k \text { value } \times 10^{-3}\left(\mathrm{~min}^{-1}\right)\end{array}$ & Regression square $\mathrm{R}^{2}$ \\
\hline \hline $20 \mathrm{mmol} / \mathrm{L}$ & 0.000996 & 0.98624 \\
$30 \mathrm{mmol} / \mathrm{L}$ & 0.001367 & 0.98408 \\
$40 \mathrm{mmol} / \mathrm{L}$ & 0.002199 & 0.96519 \\
$50 \mathrm{mmol} / \mathrm{L}$ & 0.004014 & 0.97792 \\
$60 \mathrm{mmol} / \mathrm{L}$ & 0.003048 & 0.95529 \\
$70 \mathrm{mmol} / \mathrm{L}$ & 0.001704 & 0.96581 \\
\hline
\end{tabular}

\subsubsection{Stability of the $\mathrm{ZnO} /$ zeolite $\mathrm{Na}-\mathrm{A}$ catalyst}

To test the stability of the $\mathrm{ZnO} /$ Zeolite $\mathrm{Na}-\mathrm{A}$ catalyst, 5 repetitive tests were conducted with fresh $\mathrm{MB}$ solution for sample $1.5 \% \mathrm{ZnO} / \mathrm{Zeolite} \mathrm{Na}-\mathrm{A}$ in each turn and while all the previous conditions were maintained constant. After each experiment the catalyst was filtered out, washed 3 times with distilled water and then dried at $110{ }^{\circ} \mathrm{C}$ for 12 hours before reloading it to preceding experiment. In the subsequent experiments, the catalyst demonstrated degradation constant of 5.79, 5.34, 5.20, 5.05 and $4.75\left(\mathrm{x} 10^{-3}\left(\mathrm{~min}^{-1}\right)\right)$ in the $1^{\text {st }}, 2^{\text {nd }}, 3^{\text {rd }}, 4^{\text {th }}$ and $5^{\text {th }}$ repeat experiments respectively. From this result, it is clear that the catalyst deactivation became significant as from the $4^{\text {th }}$ repeat cycle implying the $\mathrm{ZnO} /$ Zeolite Na-A catalyst portrayed high stability which is vital for reusability. The probable cause of deactivation may be due to blockages of the catalysts active sites by the adsorbed intermediates. Further, the XRD spectra measurement for used and recovered catalyst was taken and a structural comparison was made as shown in figure 15 . 


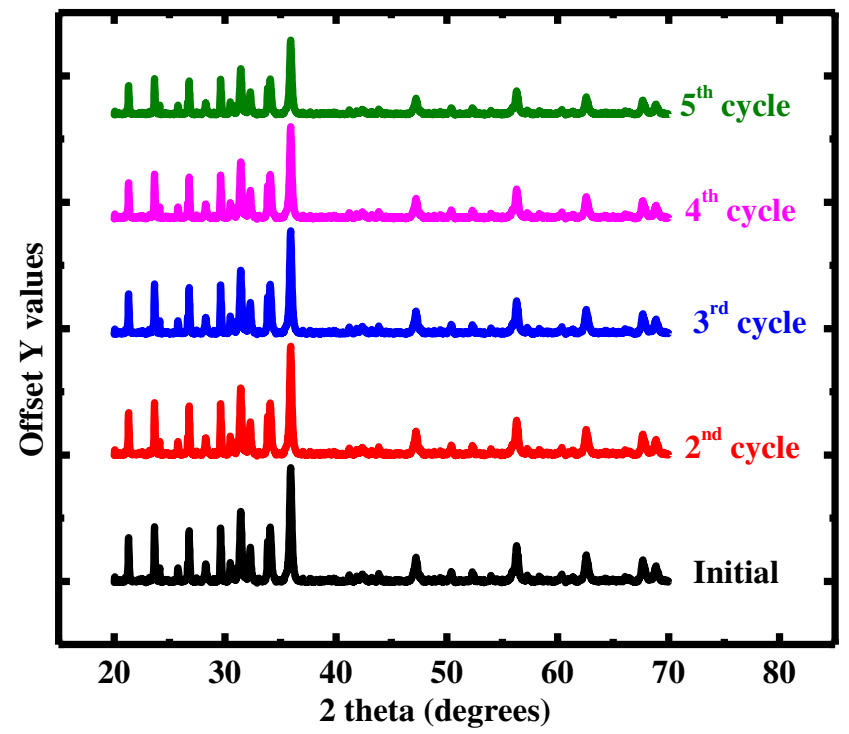

Fig. 15: XRD spectra of $1.5 \mathrm{ZnO} /$ zeolite $\mathrm{Na}-\mathrm{A}$ after the initial and repeated photodegradation of $\mathrm{MB}$

It is easily seen that the crystalline structure of the catalyst was maintained and was only weaken after several cycles. This results indicate that the $\mathrm{ZnO}$ catalysts supported by zeolite possess high levels of recyclability and therefore it can be applied in photodegradation of waste dye and generally in water treatment

\section{Conclusion}

In this study the $\mathrm{ZnO}$ was successfully loaded on the Zeolite Na-A surface and the resulting ZnO/Zeolite Na-A catalyst was characterized by XRD, SEM, TEM, UV-Visible DRS, FTIR and nitrogen adsorption-desorption techniques. The obtained results revealed that $\mathrm{ZnO}$ nanoparticles loaded on the zeolite surface were more dispersed as compared to pure $\mathrm{ZnO}$ which contributes to an increase in surface area which was also confirmed by $\mathrm{S}_{\mathrm{BET}}$ results. Increased surface area is essential for attachment of the dye molecules as well as the active species and thus increases the photodegradation rates. The highest degradation activity was obtained at $1.5 \% \mathrm{ZnO}$ loading on the zeolite and was attributed to the synergetic effects between Zeolite aluminosilicate network and the charge separation mechanism of the $\mathrm{ZnO}$ nanoparticles. Further study revealed high stability of the catalyst that is necessary for repetitive usage. It can be conclude that photodegradation of $\mathrm{ZnO}$ can be largely increased if the $\mathrm{ZnO}$ are loaded on zeolite support it is highly recommended for all de activation of dye pollutants.

\section{Conflicts of interest/Competing interests}

Authors declared no conflict of interest

\section{Funding}

The authors would like to thank the University of the Free State, Research Directorate for the research funds. 


\section{Data Availability}

Data are available from corresponding authors upon request.

\section{Code Availability}

Not applicable

\section{References}

[1] E. M. Cuerda-Correa, M. F. Alexandre-Franco and C. Fernández-González. Advanced Oxidation Processes for the removal of antibiotics from water. An overview. Water 12: (2020) 102.

[2] J. O. Tijani, O. O. Fatoba, G. Madzivire and L. F. Petrik. A review of combined Advanced Oxidation Technologies for the removal of organic pollutants from water. Water air soil pollution 225: (2014) 2102.

[3] A. Buthiyappan, A. R. A. Aziz and W. M. A. W. Daud. Recent advances and prospects of catalytic advanced oxidation process in treating textile effluents. Rev Chem Eng; 32(1): (2016) 1-47.

[4] C. B. Ong, L. Yong Ng and A. W. Mohammad. A review of $\mathrm{ZnO}$ nanoparticles as solar photocatalysts: Synthesis, mechanisms and applications. Renewable and Sustainable Energy Reviews 81: (2018) 536-551.

[5] S. W. Bian, I. A. Mudunkotuwa, T. Rupasinghe and V. H. Grassian. Aggregation and dissolution of $4 \mathrm{~nm} \mathrm{ZnO}$ nanoparticles in aqueous environments: Influence of $\mathrm{pH}$, Ionic strength, size, and adsorption of humic acid. Langmuir 27: (2011) 6059-6068.

[6] K. Pirkanniemi and M. Sillanpaa. Heterogeneous water phase catalysis as an environmental application: a review. Chemosphere 48: (2002) 1047-1060.

[7] L. V. C. Rees. Ion exchange in zeolites. Annu. Rep. Prog. Chem., Sect. A. Gen. Phys and Inorg. Chem. 67: (1970) 191-212.

[8] H. B. Hadjltaief, M. B. Zina, M. E. Galvez and P. D. Costa. Photocatalytic degradation of methyl green dye in aqueous solution over natural clay-supported $\mathrm{ZnO}^{-} \mathrm{TiO}_{2}$ catalysts. Journal of Photochemistry and Photobiology A: Chemistry 315: (2016) 25-33.

[9] R. B. Jagannatha, S. Rani and M. Padaki. ZnO zeolite nanocomposite for photocatalytic elimination of benzophenone and caffeine. ChemistrySelect 4: (2019) 1989-1993.

[10] Y. Costa-Marrero, M. B. de Andrade, J. Ellena, J. Duque-Rodríguez, T. Farías and G. Autié-Castro. Zeolite/ZnO composites based on a Cuban natural clinoptilolite and preliminary evaluation in methylene blue adsorption. Mater. Res. Express 7: (2020) 015066. 
[11] A. A. Alswata, M. B. Ahmad, N. M. Al-Hada, H. M. Kamari, M. Z. Hussein and N. A. Ibrahim. Preparation of Zeolite/Zinc Oxide Nanocomposites for toxic metals removal from water. Results in Physics 7: (2017) 723-731.

[12] H. Chen, A. Matsumoto, N. Nishimiya and K. Tsutsumi. Preparation and characterization of $\mathrm{TiO}_{2}$ incorporated Y-zeolite. Colloids and Surfaces A: Physicochemical and Engineering Aspects 157: (1999) 295-305.

[13] A. N. Ejhieh and S. Khorsandi. Photocatalytic degradation of 4-nitrophenol with ZnO supported nano-clinoptilolite zeolite. Journal of Industrial and Engineering Chemistry 20: (2014) 937-946.

[14] O. Sacco, V. Vaiano and M. Matarangolo. ZnO supported on zeolite pellets as efficient catalytic system for the removal of caffeine by adsorption and photocatalysis. Separation and Purification Technology 193: (2018) 303-310.

[15] Q. Ma, T. Fu, Y. Wan, H. Li, L. Cui and Z. Li. Development of mesoporous ZSM-5 zeolite with microporosity preservation through induced desilication. J Mater Sci 55: (2020) $11870-11890$.

[16] R. Gayatri, T. E. Agustina, D. Bahrin, R. Moeksin and G. Gustini. Preparation and characterization of ZnO-Zeolite nanocomposite for photocatalytic degradation by Ultraviolet light. Journal of Ecological Engineering 22(2): (2021) 178-186.

[17] S. Mustapha, J. O. Tijani, M. M. Ndamitso, S. A. Abdulkareem, D. T. Shuaib, A. K. Mohammed and A. Sumaila. The role of kaolin and kaolin/ZnO nanoadsorbents in adsorption studies for tannery wastewater treatment. Scientific Reports 10: (2020) 13068.

[18] J. Chen, Z. Feng, P. Ying and C. Li. ZnO clusters encapsulated inside micropores of zeolites studied by UV Raman and Laser-Induced luminescence spectroscopies. J. Phys. Chem. B, 10: (2004)12669-12676.

[19] C. Chen, Z. P. Hu, J. T. Ren, S. Zhang, Z. Wang and Z. Y. Yuan. ZnO supported on high-silica HZSM-5 as efficient catalysts for direct dehydrogenation of propane to propylene. Molecular Catalysis 476: (2019) 110508.

[20] S. Mugundan, B. Rajamannan, G. Viruthagiri, N. Shanmugam, R. Gobi and P. Praveen. Synthesis and characterization of undoped and cobalt-doped $\mathrm{TiO}_{2}$ nanoparticles via sol-gel technique. Appl Nanosci 5: (2015) 449-456.

[21] B. M. Rajbongshi, S. K. Samdarshi and B. Boro. Multiphasic bi-component $\mathrm{TiO}_{2}-\mathrm{ZnO}$ nanocomposite: synthesis, characterization and investigation of photocatalytic activity under different wavelengths of light irradiation. J Mater Sci: Mater Electron. 26: (2015) 377-384. 
[22] M. Burgos and M. Langlet. The sol-gel transformation of TIPT coatings: a FTIR study Thin Solid Films 349: (1999) 19-23.

[23] X. Cheng, X. Yu, Z. Xing and L. Yang. Synthesis and characterization of N-doped $\mathrm{TiO}_{2}$ and its enhanced visible-light photocatalytic activity 9: (2016) S1706-S1711.

[24] M. Haghigh, F. Rahmani, R. Dehghani, A. M. Tehrani and M. B. Miranzadeh. Photocatalytic reduction of $\mathrm{Cr}$ (VI) in aqueous solution over ZnO/HZSM-5 nanocomposite: optimization of $\mathrm{ZnO}$ loading and process conditions. Desalination and Water Treatment 58: (2017) 168-180.

[25] A. N. Ejhieh and F. K. Chermahini. Incorporated $\mathrm{ZnO}$ onto nanoclinoptilolite particles as the active centers in the photodegradation of phenylhydrazine. Journal of Industrial and Engineering Chemistry 20: (2014) 695-704.

[26] G. Du, P. Feng, X. Cheng, J. Li and X. Luo. Immobilizing of ZIF-8 derived ZnO with controllable morphologies on zeolite A for efficient photocatalysis. Journal of Solid State Chemistry 255: (2017) 215-218.

[27] R. Philipus, A. Noorhidayati, N. F. Djaja and R. Saleh. Comparative study of photocatalytic activity of Ni-doped $\mathrm{ZnO}$ and zeolite supported $\mathrm{Ni}$ - doped $\mathrm{ZnO}$ prepared by co-precipitation method. Materials Science Forum 827: (2015) 25-30.

[28] M. Massaro, M. Casiello, L. D'Accolti, G. Lazzara, A. Nacci, G. Nicotra, R. Noto, A. Pettignano, C. Spinella and S. Riela. One-pot synthesis of $\mathrm{ZnO}$ nanoparticles supported on halloysite nanotubes for catalytic applications. Applied Clay Science 189: (2020) 105527.

[29] A. Kocjan, M. Logar and Z. Shen. The agglomeration, coalescence and sliding of nanoparticles, leading to the rapid sintering of zirconia nanoceramics. Scientific Reports 7: 2541

[30] M. Bahrami and A. N. Ejhieh. Effect of the supported $\mathrm{ZnO}$ on clinoptilolitenanoparticles in the photodecolorization of semi-real sample bromothymol blue aqueous solution. Materials Science in Semiconductor Processing 30: (2015) 275-284.

[31] P. K. Surolia and R. V. Jasra. Photocatalytic degradation of $p$-nitrotoluene (PNT) using $\mathrm{TiO}_{2}$-modified silver-exchanged $\mathrm{NaY}$ zeolite: kinetic study and identification of mineralization pathway, Desalination and Water Treatment, 57:46 (2016) 22081-22098.

[32] A. İ. Vaizoğullar. $\mathrm{TiO}_{2} / \mathrm{ZnO}$ Supported on Sepiolite: Preparation, structural characterization, and photocatalytic degradation of flumequine. Antibiotic in Aqueous Solution, Chemical Engineering Communications 204:6 (2017) 689-697 
Figures

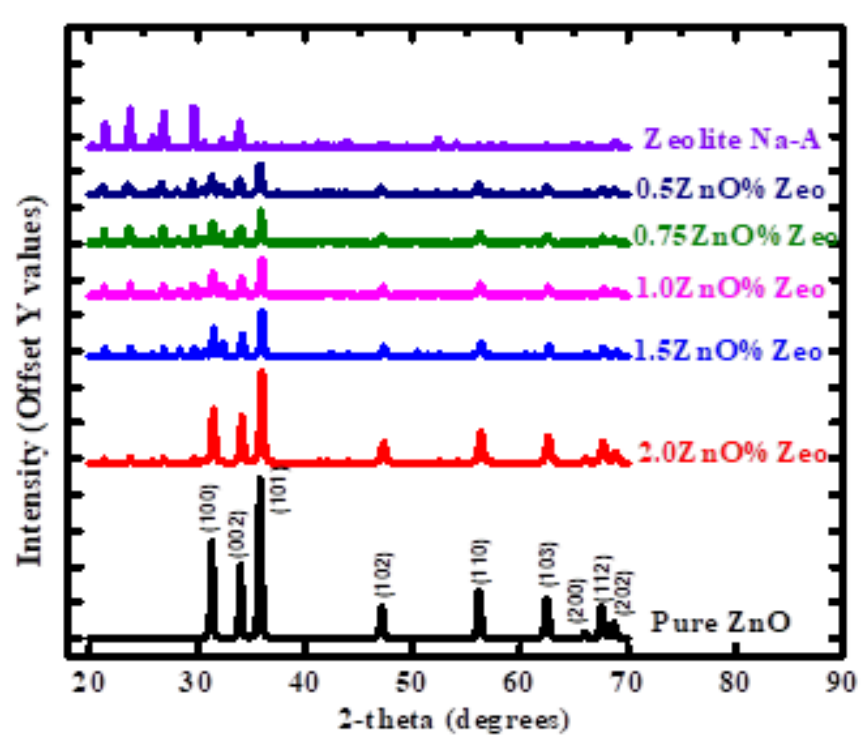

Figure 1

XRD patterns for zeolites, $\mathrm{ZnO}$ and various \%loadings of $\mathrm{ZnO} /$ Zeolite Na-A 

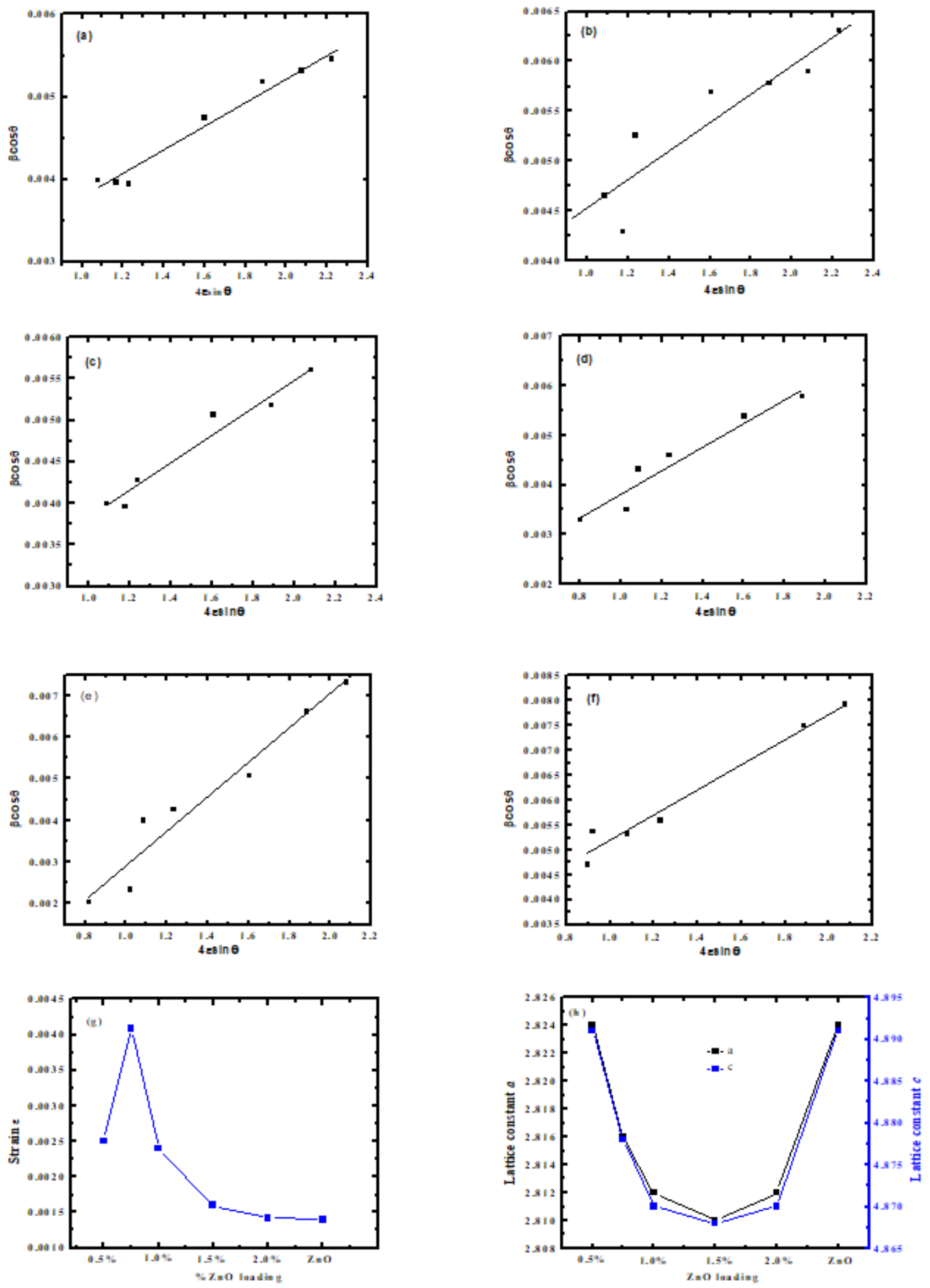

Figure 2

Evaluation of strain by Williamson-Hall plots 2(a-f) whereas $(\mathrm{g})$ and $(\mathrm{h})$ shows the relationship between microstrain and lattice constants versus $\mathrm{ZnO}$ loading respectively. 

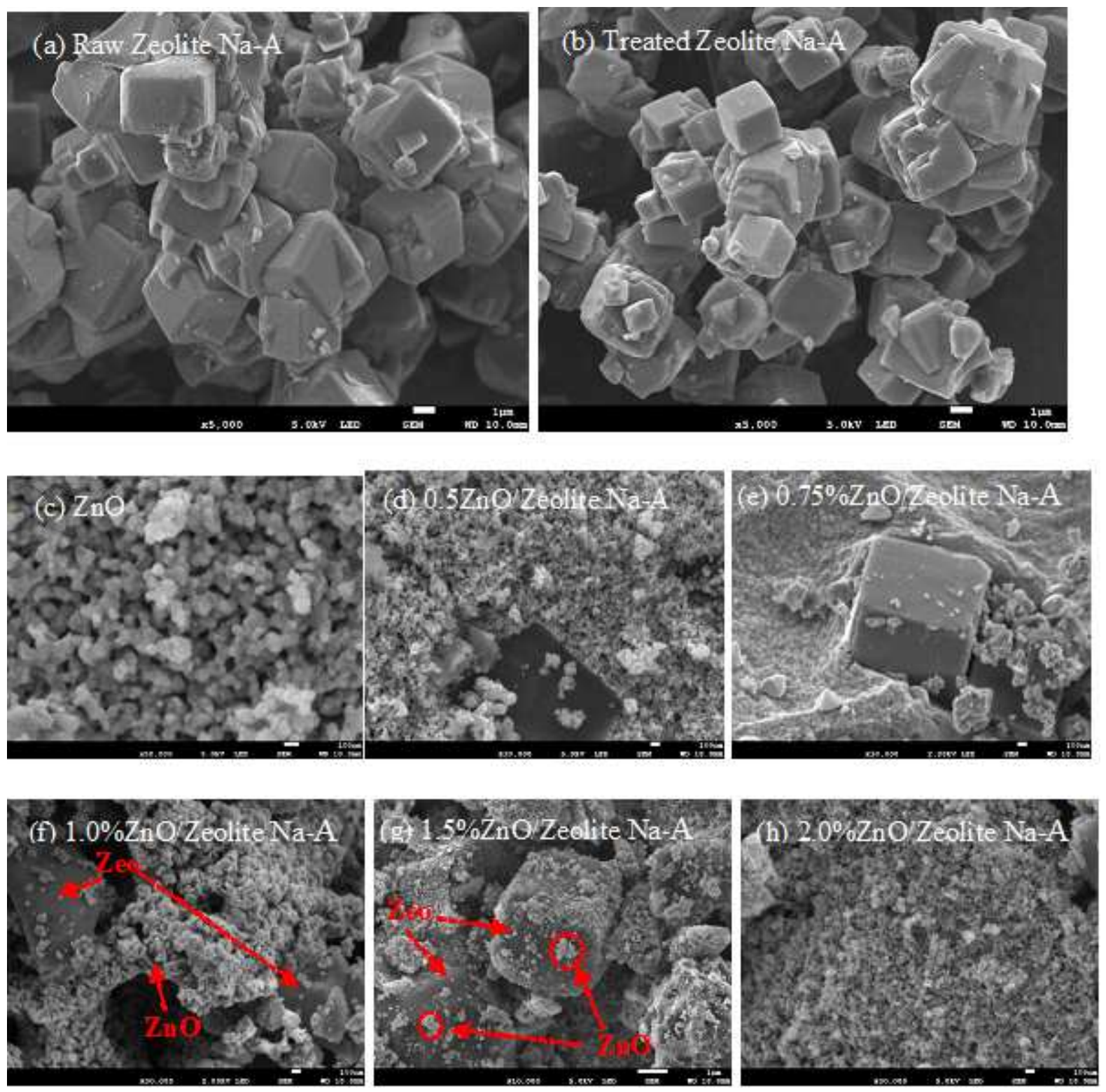

\section{Figure 3}

SEM images for different ZnO loaded zeolite Na-A 

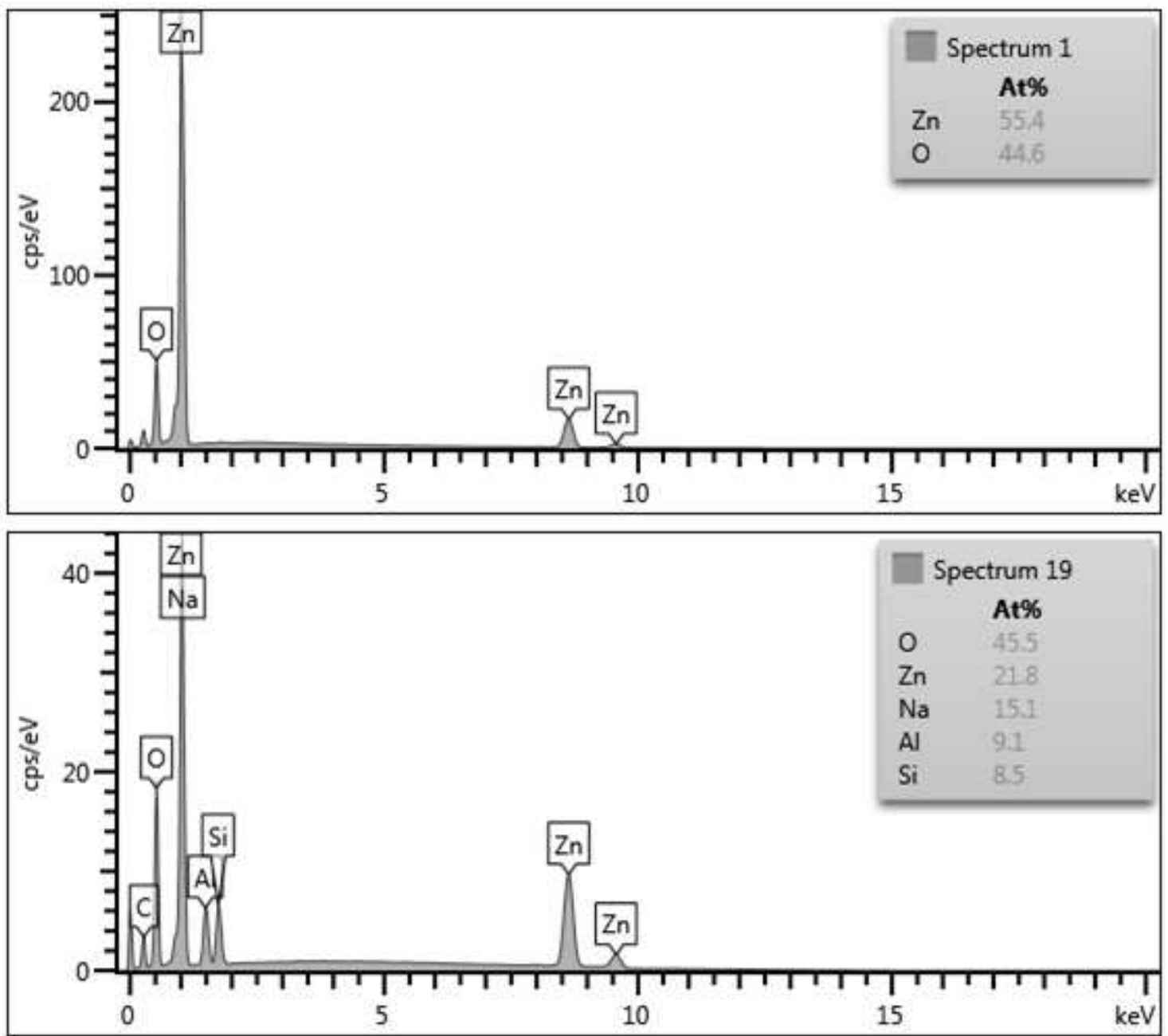

Figure 4

EDX spectra for pure ZnO (a) and ZnO/Zeolite Na-A (b) 


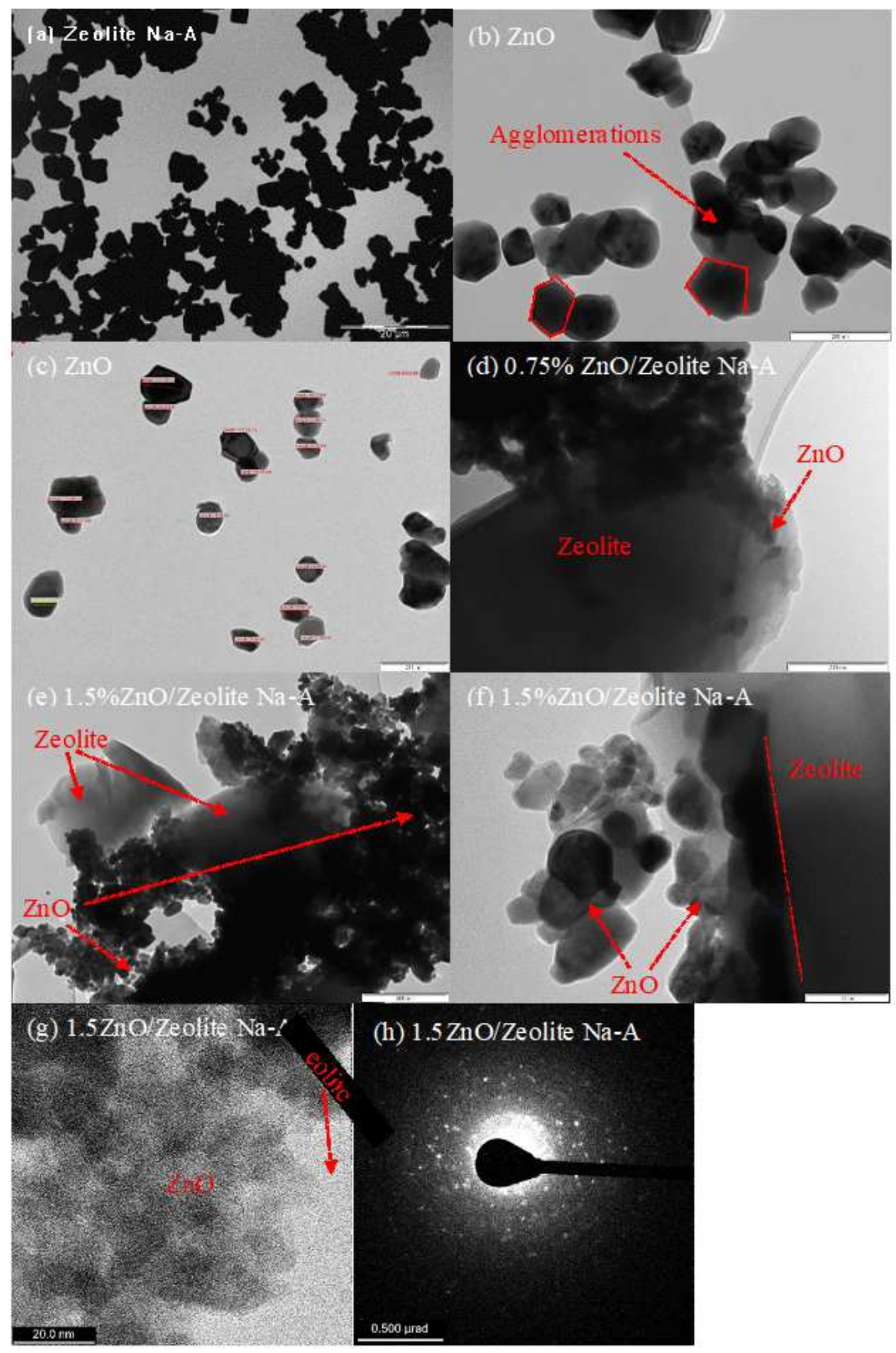

Figure 5

Selected TEM images of zeolites, ZnO and ZnO/Zeolite Na-A are shown in 5 (a-f) whereas (g) and (h) shows HRTEM image and the Selected Area Electron Diffraction SAED respectively. 

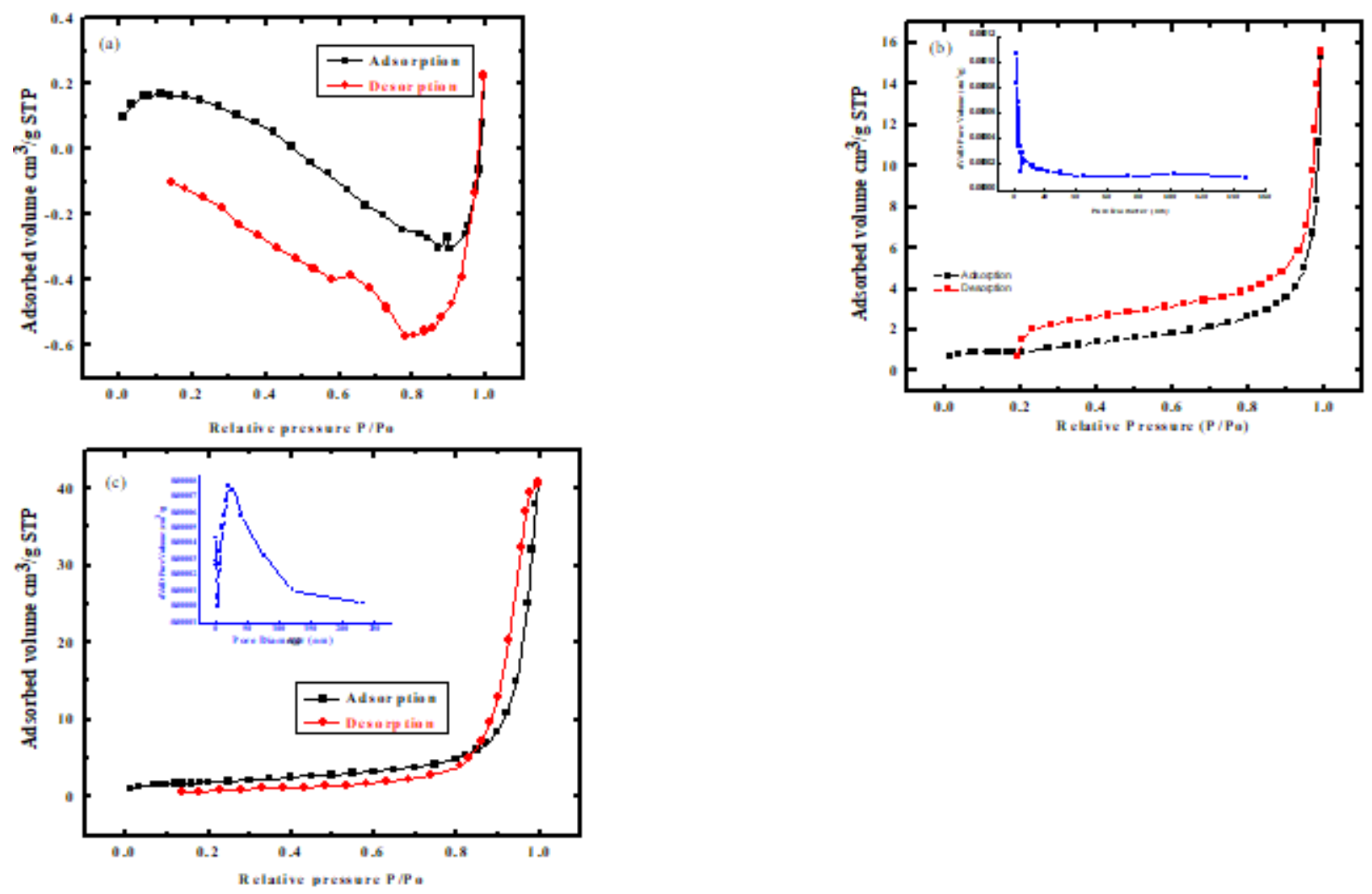

Figure 6

Nitrogen adsorption-desorption curves for pure zeolite (a), pure ZnO (b) and 1.5\%ZnO/Zeolite Na-A (c) nanopowders. Inset plots shows the respective pore size distribution.
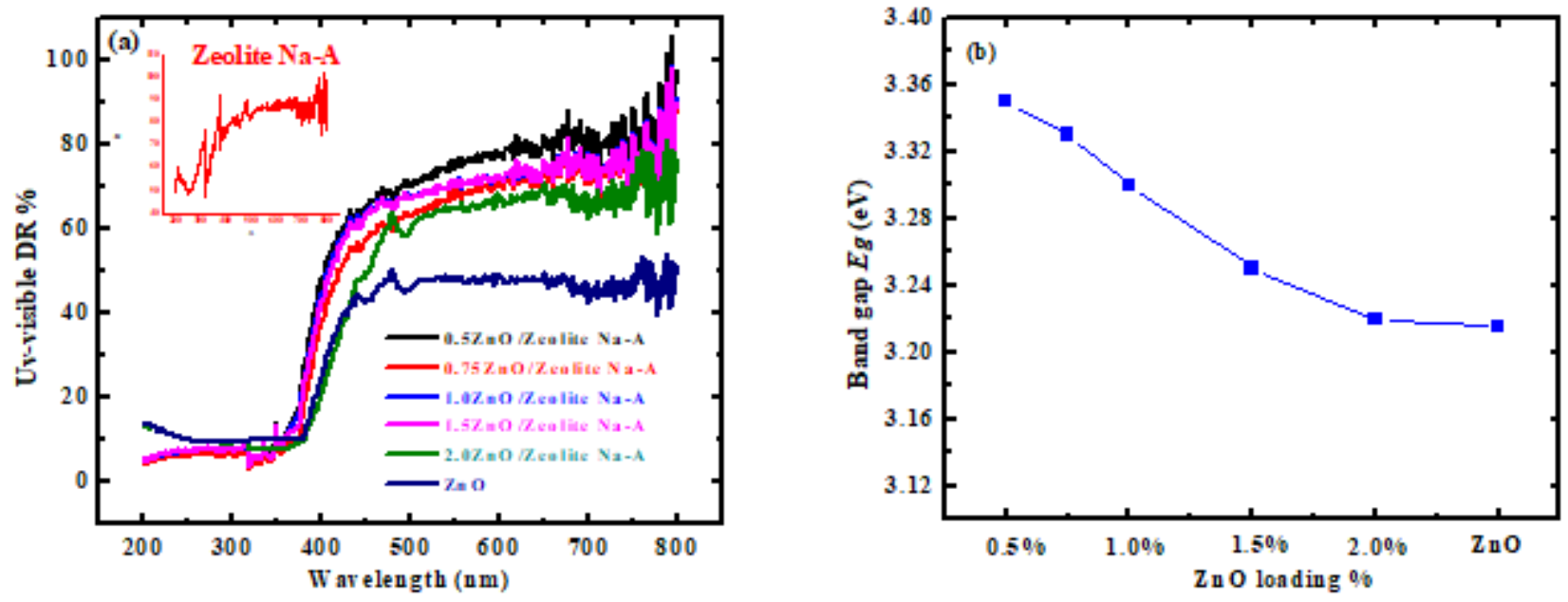

Figure 7

Diffuse reflectance spectroscopy (DRS) of pure Zeolite, pure ZnO and the ZnO/Zeolite Na-A loaded catalyst. 


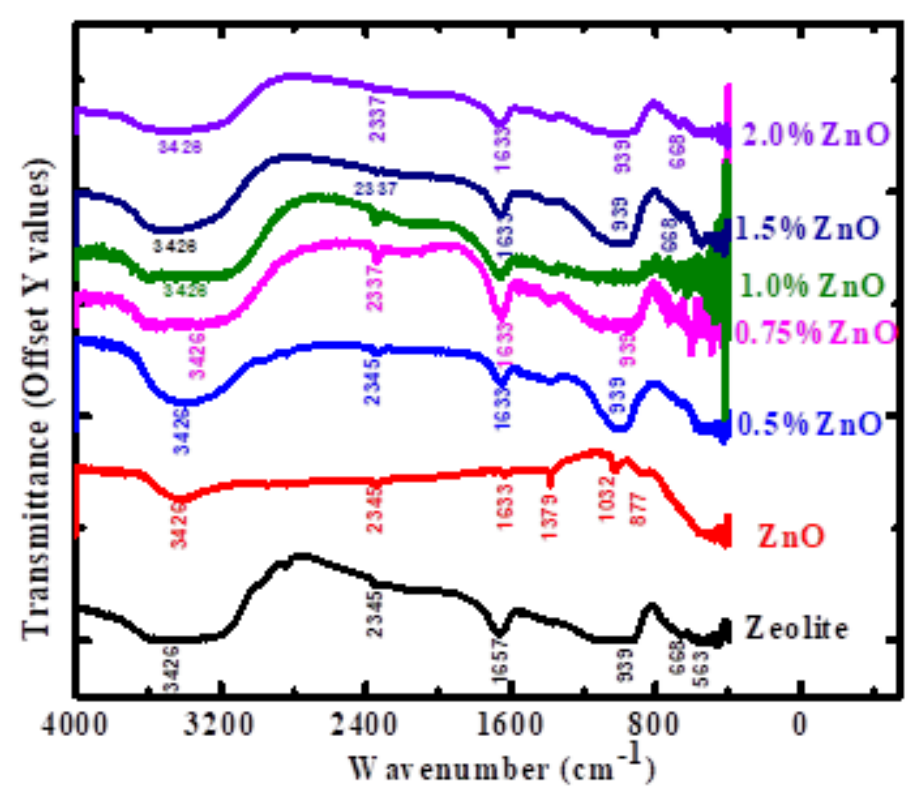

Figure 8

FTIR spectra of indicating various functional groups on Zeolite, pure ZnO and ZnO/Zeolite Na-A
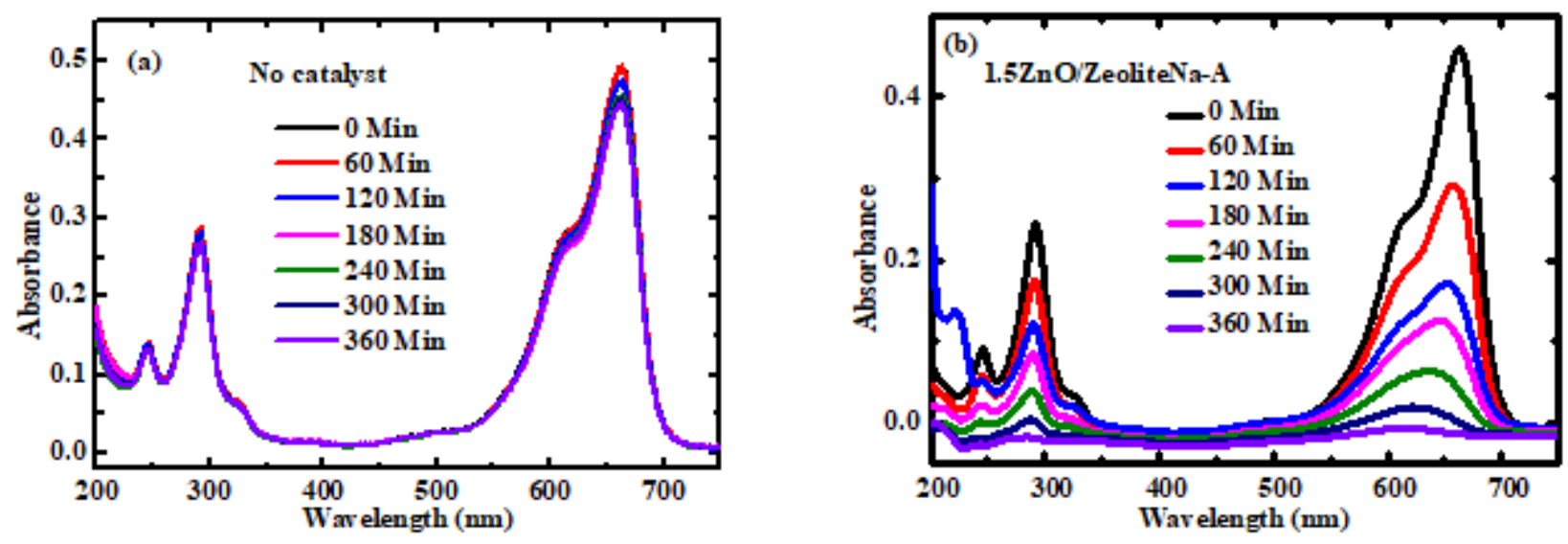

Figure 9

Effects of change of methylene blue dye peak with time on exposure to UV radiation (a) blank (b) with catalyst 

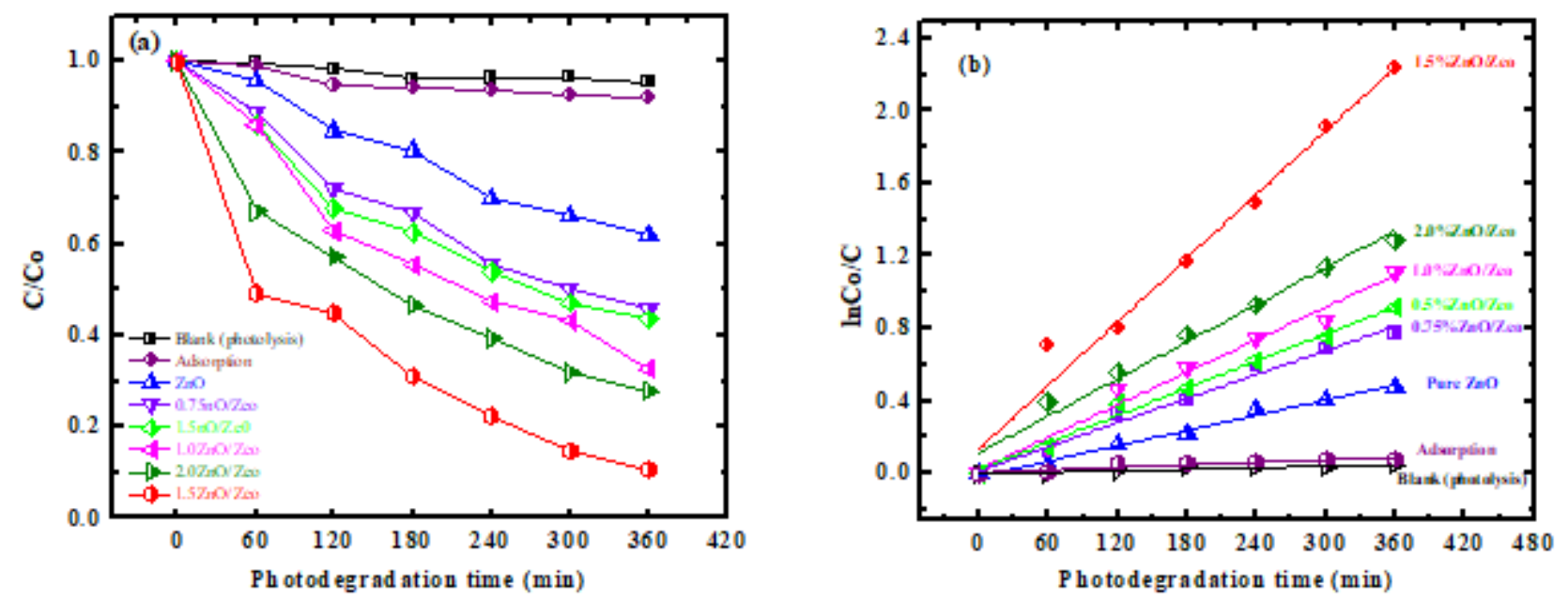

Figure 10

(a) $\mathrm{C} / \mathrm{Co}$ versus degradation time (b) the various degradation rates for different $\mathrm{ZnO}$ loading levels.

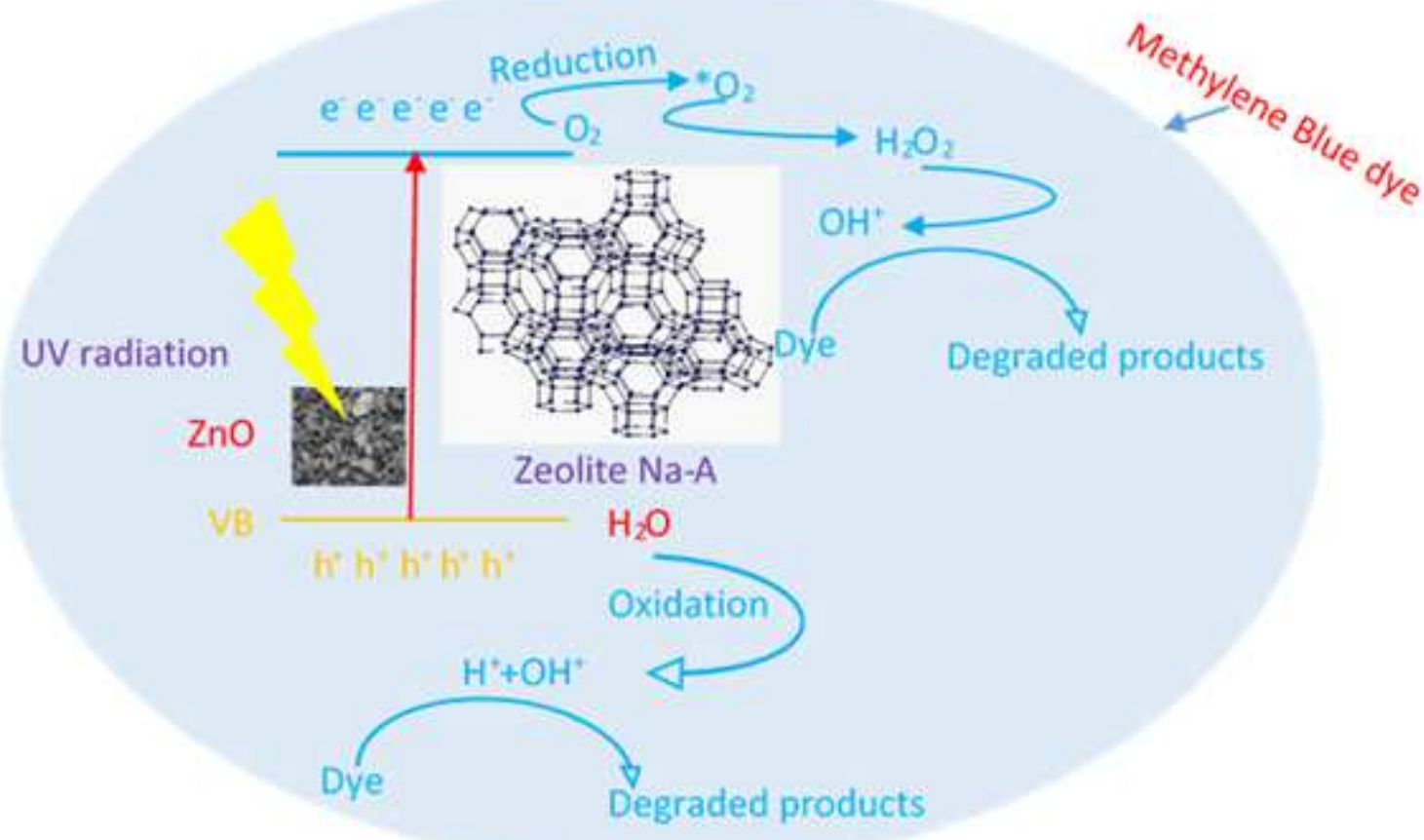

Figure 11

Photodegradation mechanism 


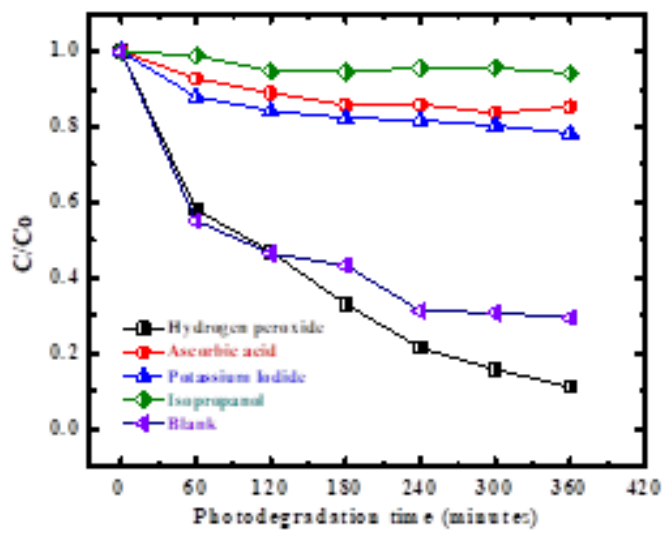

Figure 12

Effect of different radical scavengers and oxidant

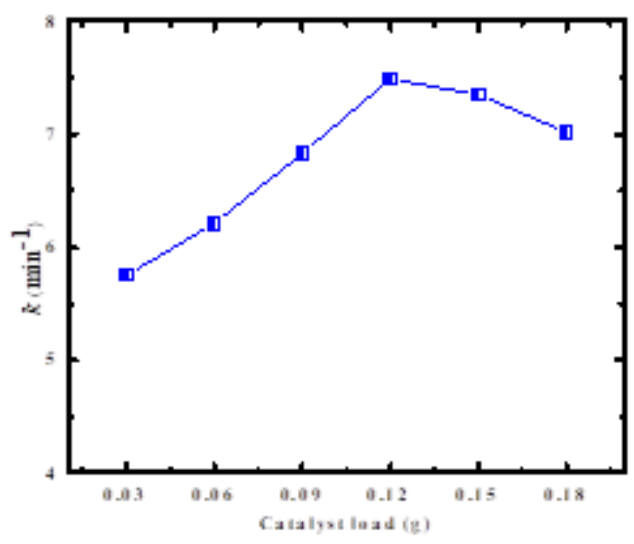

Figure 13

Variation between degradation constant $\mathrm{k}$ against $\mathrm{ZnO} /$ Zeolite $\mathrm{Na}-\mathrm{A}$ catalyst loading
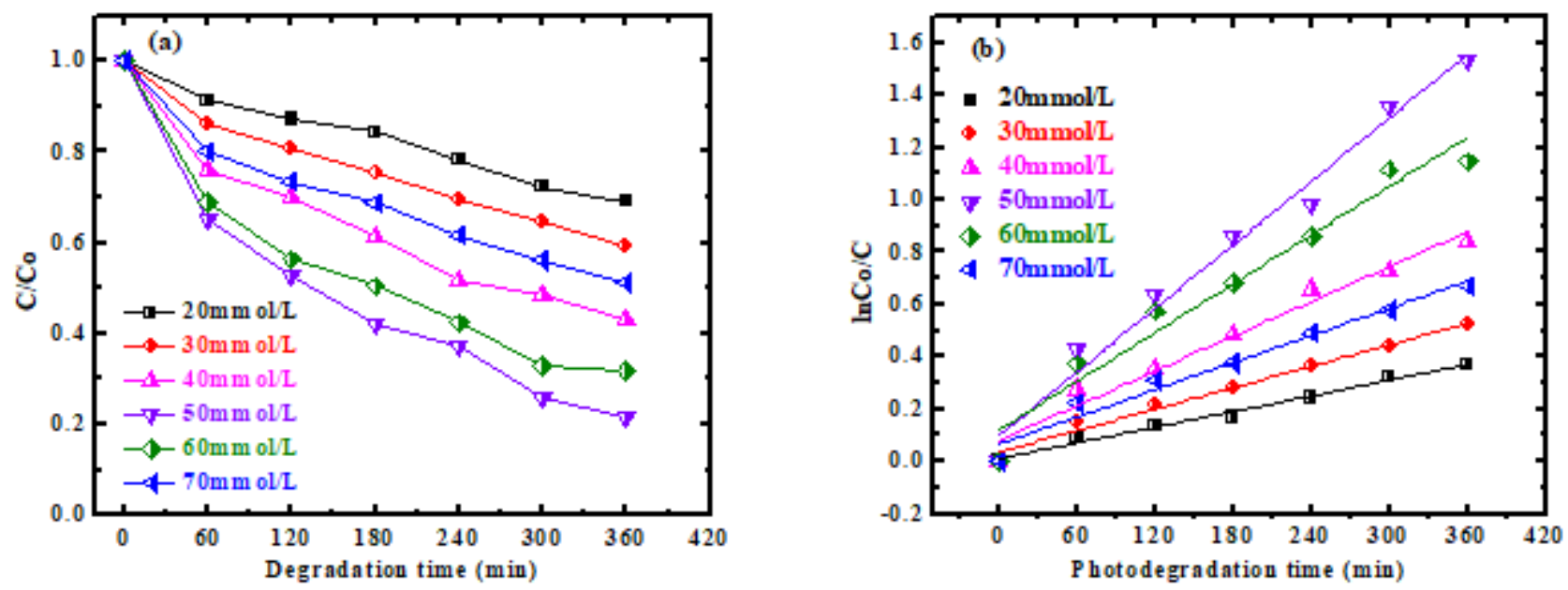

Figure 14

(a) $\mathrm{C} /$ Co versus degradation time at differenet $\mathrm{MB}$ concentarations (b) the various degradation rates for at different MB concentrations. 


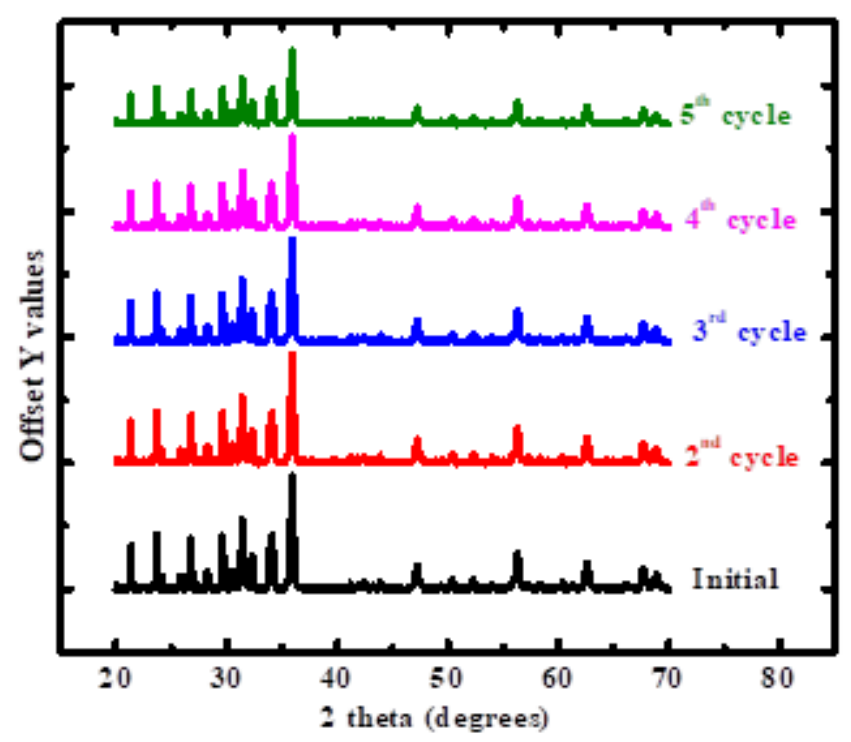

Figure 15

XRD spectra of $1.5 \mathrm{ZnO} /$ zeolite $\mathrm{Na}-\mathrm{A}$ after the initial and repeated photodegradation of $\mathrm{MB}$ 